\title{
Homogeneous photometry VII. Globular clusters in the Gaia era.
}

\author{
P. B. Stetson ${ }^{1 \star}$, E. Pancino ${ }^{2,3}$, A. Zocchi ${ }^{4}$, N. Sanna ${ }^{2}$, M. Monelli ${ }^{5,6}$ \\ ${ }^{1}$ Herzberg Astronomy and Astrophysics, National Research Council, 5071 West Saanich Road, Victoria, British Columbia V9E 2E7, Canada \\ ${ }^{2}$ INAF - Osservatorio Astrofisico di Arcetri, Largo Enrico Fermi 5, I-50125 Firenze, Italy \\ ${ }^{3}$ Space Science Data Center, ASI, via del Politecnico snc, I-00133 Roma, Italy \\ ${ }^{4}$ European Space Research and Technology Centre (ESA/ESTEC), Keplerlaan 1, 2201 AZ Noordwijk, The Netherlands \\ ${ }^{5}$ Instituto de Astrofísica de Canarias, Calle Via Lactea, E-38205, La Laguna, Tenerife, Spain \\ ${ }^{6}$ Universidad de La Laguna, Dpto. Astrofísica, E-38206, La Laguna, Tenerife, Spain
}

Accepted XX month XX. Received YY month YY; in original form ZZ month ZZ

\begin{abstract}
We present wide-field, ground-based Johnson-Cousins UBVRI photometry for 48 Galactic globular clusters based on almost 90000 public and proprietary images. The photometry is calibrated with the latest transformations obtained in the framework of our secondary standard project, with typical internal and external uncertainties of order a few millimagnitudes. These data provide a bridge between existing small-area, high-precision HST photometry and all sky-catalogues from large surveys like Gaia, SDSS, or LSST. For many clusters, we present the first publicly available photometry in some of the five bands (typically $U$ and $R$ ). We illustrate the scientific potential of the photometry with examples of surface density and brightness profiles and of colour-magnitude diagrams, with the following highlights: (i) we study the morphology of NGC 5904, finding a varying ellipticity and position angle as a function of radial distance; (ii) we show $U$-based colour-magnitude diagrams and demonstrate that no cluster in our sample is free from multiple stellar populations, with the possible exception of a few clusters with high and differential reddening or field contamination, for which more sophisticated investigations are required. This is true even for NGC 5694 and Terzan 8 , that were previously considered as (mostly) single-population candidates.
\end{abstract}

Key words: standards - techniques: photometric - catalogues - globular clusters: general

\section{INTRODUCTION}

Star clusters, and especially globular clusters (GCs), have always been key objects: witnesses to the initial epochs of star formation in the Universe, exceptional dynamical laboratories, and test benches for stellar structure and evolution theories. For decades, the traditional simple understanding of GCs considered them to be spherically symmetric, isotropic, non-rotating stellar systems, each made of a single homogeneous stellar population. This situation evolved following the technological revolution started in the 1980's - chargecoupled device (CCD) cameras on small and large telescopes, high-resolution and multi-object spectrographs, astronomical data archives - that has provided a huge amount of information, often of exquisite quality.

^ E-mail:Peter.Stetson@nrc-cnrc.gc.ca
Deviations from spherical symmetry have been detected for many Galactic (Geyer, Hopp \& Nelles 1983; White \& Shawl 1987; Chen \& Chen 2010) and extra-Galactic (Elson, Fall \& Freeman 1987; Davoust \& Prugniel 1990; Han \& Ryden 1994; Barmby et al. 2007; van den Bergh 2008; Wang \& Ma 2013) GCs, motivating a treatment of these systems that takes into account their flattening, and demanding that the cause of the observed morphology be determined. Rotation both in the plane of the sky (Bellini et al. 2017; Gaia Collaboration et al. 2018; Bianchini et al. 2018; Milone et al. 2018) and along the line of sight (e.g., see Fabricius et al. 2014; Boberg et al. 2017; Cordero et al. 2017; Lanzoni et al. 2018), pressure anisotropy (van Leeuwen et al. 2000; Watkins et al. 2015), and effects due to interaction with the tidal field of the host galaxy (Drukier et al. 1998; Küpper et al. 2010; Da Costa 2012) have also been detected in many GCs, pointing out the need for a more realistic description of their dynamics that would allow us to finally assess the formation 
Table 1. Summary of facilities used, with the range of years covered by the observations and the total number of images collected in each photometric band, ( $\mathrm{n}_{o}$ are non-standard filters). More details, including the full image credits, can be found in Appendix A.

\begin{tabular}{|c|c|c|c|c|c|c|c|c|c|}
\hline Site & Telescope & Instrument & Dates & $\mathrm{n}_{U}$ & $\mathrm{n}_{B}$ & $\mathrm{n}_{V}$ & $\mathrm{n}_{R}$ & $\mathrm{n}_{I}$ & $\mathrm{n}_{o}$ \\
\hline Apache Point & ARCSAT $0.5 \mathrm{~m}$ & APOGEE & 2017 & 0 & 12 & 22 & 0 & 1 & 0 \\
\hline Apache Point & APO $3.5 \mathrm{~m}$ & Arctic & 2017 & 0 & 10 & 12 & 0 & 0 & 0 \\
\hline BNAO Rohzen & BNAO $2.0 \mathrm{~m}$ & Photometrics CE200A & 2001 & 0 & 212 & 213 & 2 & 168 & 0 \\
\hline Cerro Pachón & SOAR $4.1 \mathrm{~m}$ & SOI & 2008-2012 & 276 & 254 & 288 & 144 & 504 & 534 \\
\hline Cerro Tololo & CTIO $0.9 \mathrm{~m}$ & TI1/2, Tek, Tek2k, RCA & 1986-2012 & 39 & 2377 & 2630 & 416 & 930 & 876 \\
\hline Cerro Tololo & CTIO $1.0 \mathrm{~m}$ & Y4KCam & 2006-2013 & 29 & 2144 & 2654 & 349 & 1110 & 1362 \\
\hline Cerro Tololo & CTIO 1.3m & ANDICAM & $2005-2008$ & 0 & 270 & 294 & 0 & 257 & 0 \\
\hline Cerro Tololo & CTIO 1.5m & TI1/2, Tek, Tek2k, Site2K & 1987-1998 & 16 & 234 & 234 & 20 & 143 & 0 \\
\hline Cerro Tololo & CTIO $4.0 \mathrm{~m}$ & TI1/2, Mosaic1/2, Tek2k, DECam & $1983-2016$ & 457 & 1325 & 1688 & 178 & 1838 & 2958 \\
\hline DAO Victoria & DAO $1.8 \mathrm{~m}$ & SITE1 & 1995-1996 & 0 & 7 & 12 & 8 & 8 & 0 \\
\hline ESO La Silla & Dutch $0.9 \mathrm{~m}$ & Tektronic 33 & 1997 & 0 & 0 & 167 & 0 & 166 & 0 \\
\hline ESO La Silla & Danish $1.5 \mathrm{~m}$ & MAT/EEV, DFOSC & 1995-2005 & 8 & 2689 & 5079 & 2114 & 890 & 117 \\
\hline ESO La Silla & $\mathrm{ESO} / \mathrm{MPI} 2.2 \mathrm{~m}$ & WFI & 1999-2012 & 2456 & 4000 & 5072 & 1096 & 3647 & 184 \\
\hline ESO La Silla & NTT $3.6 \mathrm{~m}$ & EMMI, SUSI, EFOSC & $1992-2014$ & 184 & 1991 & 1159 & 321 & 819 & 108 \\
\hline ESO Paranal & VLT $8.0 \mathrm{~m}$ & VIMOS, FORS1, FORS2 & 1999-2012 & 200 & 304 & 692 & 243 & 306 & 0 \\
\hline Kitt Peak & KPNO 0.9m & $\mathrm{RCA}, \mathrm{T} 2 \mathrm{kA}$ & 1984-1999 & 0 & 179 & 188 & 0 & 93 & 92 \\
\hline Kitt Peak & KPNO $2.1 \mathrm{~m}$ & CCD, T1kA & 1992-1994 & 0 & 1 & 74 & 1 & 77 & 0 \\
\hline Kitt Peak & WIYN 3.5m & WIYNMiniMos & 2001-2014 & 0 & 0 & 12 & 18 & 30 & 18 \\
\hline Kitt Peak & KPNO $4.0 \mathrm{~m}$ & RCA, Mosaic1, T2kB & $1984-2011$ & 0 & 213 & 231 & 48 & 180 & 2075 \\
\hline La Palma & JKT $1.0 \mathrm{~m}$ & GEC, EEV, SITE, TEK & 1991-2003 & 5 & 137 & 240 & 118 & 190 & 2 \\
\hline La Palma & INT $2.5 \mathrm{~m}$ & GEC, EEV, Tek, WFC, Patterson & 1986-2018 & 1157 & 3129 & 2575 & 852 & 2344 & 320 \\
\hline La Palma & NOT $2.6 \mathrm{~m}$ & CCD7 & 1993-2001 & 0 & 45 & 78 & 0 & 86 & 0 \\
\hline La Palma & TNG 3.6m & OIG, GEC, E2V, Loral & 1990-2009 & 26 & 113 & 176 & 0 & 97 & 0 \\
\hline Las Campanas & Warsaw $1.3 \mathrm{~m}$ & 8k MOSAIC & 2003-2008 & 0 & 983 & 1077 & 0 & 112 & 0 \\
\hline Las Campanas & Magellan $6.5 \mathrm{~m}$ & MagIC, IMACS & 2006-2009 & 0 & 91 & 7359 & 0 & 128 & 0 \\
\hline MMO Nantucket & MMO $0.4 \mathrm{~m}$ & Roper PVCAM & 2014 & 0 & 0 & 146 & 145 & 0 & 0 \\
\hline Mauna Kea & $\mathrm{UH} 2.2 \mathrm{~m}$ & CCD & 1986 & 0 & 2 & 2 & 1 & 3 & 0 \\
\hline Mauna Kea & CFHT $3.6 \mathrm{~m}$ & Lick, HRCam, CFH12K, MegaPrime & $1984-2014$ & 5 & 736 & 1208 & 383 & 764 & 520 \\
\hline Mauna Kea & Subaru $8.2 \mathrm{~m}$ & SuprimeCam & 2008 & 0 & 250 & 270 & 0 & 0 & 0 \\
\hline McDonald & LCOGT $1.0 \mathrm{~m}$ & CCD camera & 2013 & 0 & 0 & 120 & 0 & 109 & 0 \\
\hline Siding Spring & LCOGT $1.0 \mathrm{~m}$ & CCD camera & 2014 & 0 & 0 & 28 & 0 & 22 & 0 \\
\hline Siding Spring & AAT $3.9 \mathrm{~m}$ & CCD1 & 1991 & 0 & 6 & 0 & 28 & 0 & 0 \\
\hline Sutherland & SAAO $1.0 \mathrm{~m}$ & CCD 986 & 2006 & 56 & 56 & 68 & 1 & 70 & 0 \\
\hline Sutherland & LCOGT $1.0 \mathrm{~m}$ & CCD camera & 2013-2014 & 0 & 0 & 449 & 0 & 380 & 0 \\
\hline San Pedro Mártir & SPM $0.84 \mathrm{~m}$ & $\mathrm{E} 2 \mathrm{~V}-4290$ & 2014-2018 & 19 & 631 & 28 & 28 & 29 & 0 \\
\hline San Pedro Mártir & SPM $2.14 \mathrm{~m}$ & E2V-4290 & 2018 & 9 & 13 & 13 & 14 & 14 & 0 \\
\hline Teide & IAC80 0.8m & CAMELOT & $2017-2018$ & 22 & 37 & 31 & 28 & 31 & 0 \\
\hline
\end{tabular}

mechanism of these systems and to fully understand their evolution. The ESA space mission Gaia (Gaia Collaboration et al. 2016b), in particular, by providing access to the full six-dimensional phase space of positions and velocities for large numbers of stars in clusters, will finally allow us to probe the full complexity of their structural, kinematic, and chemical properties through time.

Moreover, GCs are now known to host Multiple Populations (MPs) (Kraft 1994; Gratton, Carretta \& Bragaglia 2012; Bastian \& Lardo 2018). The first convincing evidence for MPs comes from abundance differences among their stars, in particular anti-correlations in a set of light elements $(\mathrm{C}, \mathrm{N}, \mathrm{O}$, and $\mathrm{Na}$; often also $\mathrm{Mg}, \mathrm{Al}, \mathrm{Li}$, and $\mathrm{F}$ ) that have been found in GCs with adequate data. Generally, these variations are not accompanied by a net chemical evolution: heavy elements and the total $\mathrm{C}+\mathrm{N}+\mathrm{O}$ abundance are constant in most GCs, except for a minority $(\simeq 20 \%$, see Milone et al. 2017) of anomalous GCs (see also Section 5.3). The probable origin of these variations is found in the CNO cycle, converting hydrogen into helium, with its hotter Ne$\mathrm{Na}$ and $\mathrm{Mg}-\mathrm{Al}$ sub-cycles (Denisenkov \& Denisenkova 1989;
Kraft 1994). Unfortunately, even the most successful scenarios proposed so far (Decressin et al. 2007; D'Ercole et al. 2008) appear to suffer from major drawbacks (Bastian et al. 2013; Renzini et al. 2015), and the problem can be considered unsolved at the present time. Many spectroscopic investigations have explored the chemistry of GCs in great detail, and large spectroscopic surveys such as APOGEE (Mészáros et al. 2015) and Gaia-ESO (Pancino et al. 2017) are complementing the limited spectroscopic capabilities of Gaia for abundance determinations.

The complex chemistry of GCs is reflected in their photometric properties. Multiple evolutionary sequences have been observed in some colour-magnitude diagrams (CMDs), starting with the ground-based discovery of the anomalous red giant branch (RGB) in $\omega$ Cen (Lee et al. 1999; Pancino et al. 2000). Since then, space-based imagery has made it possible to routinely obtain exquisitely fine details in the colour-magnitude and colour-colour diagrams of most globular clusters (Piotto et al. 2015; Milone et al. 2017): multiband photometry involving ultraviolet filters is especially effective thanks to the $U$-band's sensitivity to $\mathrm{CN}$ abundance, 
allowing us to study the relative behaviour, proportion, and distribution of the MPs (Marino et al. 2008; Lardo et al. 2011; Sbordone et al. 2011; Milone et al. 2017).

However, space-based photometry, which is the ideal means to study the crowded central regions of GCs, typically covers a relatively small area, generally amounting to no more than one or two half-light radii, depending on the GC. On the other hand Gaia - which offers extremely precise and accurate photometry over the whole sky - only contains three broad-band colours $\left(G, G_{B P}\right.$, and $\left.G_{R P}\right)$, that are not able to separate MPs. Finally, large surveys like SDSS (Abolfathi et al. 2018), which offer multiband, all-sky photometry and could nicely complement space-based photometry, have not employed the point-spread-function (PSF) fitting techniques that are required to obtain the best results in crowded stellar fields (An et al. 2008).

The goal of the present study is therefore to provide multiband, wide-field, ground-based photometry to complement the upcoming data from Gaia and large photometric and spectroscopic surveys, based on a large collection of publicly available and proprietary images accumulated by the first author over many years. By bridging the gap between the space-based photometry of GC centres and the large surveys covering their outer parts, this catalogue will allow for a complete coverage of the extent of these systems, making it possible to study them with unprecedented detail.

This paper is organized as follows: in Section 2 we describe the sample selection, data sources, and image prereductions; in Section 3 we describe the photometric measurement and calibration process; in Section 4 we illustrate the final catalogue content; in Section 5 we present some preliminary results; in Section 6 we summarize our findings and draw conclusions.

\section{SAMPLE AND DATA REDUCTIONS}

The photometry presented in this paper is based on a large data collection ${ }^{1}$, containing more than half a million astronomical images obtained with CCDs for popular science targets like dwarf galaxies, open clusters, globular clusters, supernova hosts, photometric standard fields, and other targets of wide astronomical interest (Stetson 2009). The majority of these images are public-domain data downloaded from astronomical data archives, although there are also some proprietary images obtained by ourselves or donated by colleagues. Some of these latter date back to the days before astronomical archives existed: the earliest CCD images in the data set are from January 8, 1983 (see Table 1 and Appendix A), and we continue to accumulate data even now.

PSF photometry is extracted from these digital images with DAOPHOT and ALLFRAME, and is aperturecorrected and merged using the associated suite of ancillary software packages (Stetson 1987, 1992, 1994). The resulting photometry in the UBVRI filters is homogeneously calibrated to the photometric system defined by Landolt (1992) employing colour transformations optimized independently

\footnotetext{
${ }^{1}$ http://www3.cadc-ccda.hia-iha.nrc-

cnrc.gc.ca/en/community/STETSON/index.html
}

for each observing run, each night, and each CCD used for observation. In the process we define a large selection of secondary photometric standard stars in the UBVRI filters to help register the many different observing runs to a common photometric system. Some results from the collection have already been published for dwarf galaxies (Smecker-Hane et al. 1994; Stetson, Hesser \& Smecker-Hane 1998; Stetson et al. 2014), GCs (Stetson 2005; Stetson, Catelan \& Smith 2005; Bergbusch \& Stetson 2009; Viaux et al. 2013), and Open Clusters (OCs, Stetson, Bruntt \& Grundahl 2003; Brogaard et al. 2012), particularly in the "Homogeneous photometry" series of papers.

The database is constantly evolving with the addition of new images, the definition of new secondary standards, and the refinement of the photometric indices for existing standards. Occasionally a star that has been used as a standard is discarded as evidence of intrinsic variability becomes significant. Thus the photometric catalogues published by us here and elsewhere are subject to revision over time, but this takes the form of incrementally improved precision and accuracy in the published photometric indices. Constant referral back to Landolt's original photometric results (Landolt 1992) safeguards against secular drift of our photometric system over time. In any given publication, the results we present are the best we can do at that particular time and we make every attempt to provide an objective description of the instantaneous quality of the data. So far, none of the claimed results of the previous studies have been challenged in any way by our subsequent improvements in the data.

Here we present photometry for 48 GCs (Section 2.1) obtained from the current image collection (Section 2.2) using the latest photometric solutions (Section 3.1). The present catalogues supersede previous publications, such as those for 47 Tuc by Bergbusch \& Stetson (2009), for M 15 by Stetson (1994), or for the GC collection in the SUMO project (Monelli et al. 2013). The previously published results are fully consistent, within the claimed uncertainties, with those presented here.

The major strengths of these catalogues are the wide field of view, the large number of images used, the multiband optical $U B V R I$ photometry, and the robust, precise photometric calibrations based, typically, on hundreds of photometric standard-star observations in each filter on each CCD on each night. To illustrate: the current data set contains some 7508 night-chip-filters of calibrated observations. Among these, the median number of photometric standard stars observed per night per chip per filter is 1236 .

For the central regions of the target clusters the groundbased photometry presented here has less precision and less depth than space-based photometry (Sarajedini et al. 2007; Piotto et al. 2015) due to stellar crowding in the central parts. On the other hand, due to the use of the somewhat more venerable $U B V R I$ bandpasses, our results may be more directly and accurately compared to many legacy studies in the historical literature. Furthermore, it would require only minor effort to find stars in common between our catalogues and the published space-based catalogues to establish relative transformations and merge the datasets. We have not done that here because it is not essential for our immediate purposes and would unnecessarily expand this article. 
Table 2. For the GC sample considered in this paper, we list our new central coordinates (Section 4.2); the metallicity [Fe/H], reddening $\mathrm{E}(\mathrm{B}-\mathrm{V})$, total luminosity $\mathrm{M}_{\mathrm{V}}^{\text {tot }}$, and apparent visual distance modulus $(\mathrm{M}-\mathrm{m})_{\mathrm{V}}$ from the Harris (2010) catalogue; the total number of images analyzed per GC ( $\mathrm{n}_{\text {images }}$ ); the number of RR Lyrae variables catalogued per GC $\left(\mathrm{n}_{\mathrm{RR}}\right)$; the $\mathrm{X}_{0}$ and $\mathrm{Y}_{0}$ GC centroids (Section 4.2); and the priority ranking based on the criteria described in Section 2.1.

\begin{tabular}{|c|c|c|c|c|c|c|c|c|c|c|c|c|}
\hline Cluster & & $\begin{array}{r}\mathrm{RA} \\
(\mathrm{hh} \mathrm{mm} \mathrm{ss})\end{array}$ & $\begin{array}{r}\text { Dec } \\
(\mathrm{dd} \mathrm{mm} \mathrm{ss})\end{array}$ & $\begin{array}{r}{[\mathrm{Fe} / \mathrm{H}]} \\
(\mathrm{dex})\end{array}$ & $\begin{array}{r}\mathrm{E}(\mathrm{B}-\mathrm{V}) \\
(\mathrm{mag})\end{array}$ & $\begin{array}{c}\mathrm{M}_{\mathrm{V}}^{\text {tot }} \\
(\mathrm{mag})\end{array}$ & $\begin{array}{r}(\mathrm{M}-\mathrm{m})_{\mathrm{V}} \\
(\mathrm{mag})\end{array}$ & $\mathrm{n}_{\text {images }}$ & $\mathrm{n}_{\mathrm{RR}}$ & $\begin{array}{r}\mathrm{X}_{0} \\
\text { (pix) }\end{array}$ & $\begin{array}{r}\mathrm{Y}_{0} \\
\text { (pix) }\end{array}$ & Rank \\
\hline NGC 104 & 47 Tuc & 002403.63 & -720446.6 & -0.76 & 0.04 & -9.42 & 13.37 & 2553 & 2 & +303 & +55 & 1 \\
\hline NGC 288 & & 005244.98 & -263504.8 & -1.24 & 0.03 & -6.74 & 14.83 & 1178 & 2 & +235 & -444 & 21 \\
\hline NGC 1261 & & $\begin{array}{lll}03 & 12 & 15.97\end{array}$ & -551257.6 & -1.35 & 0.01 & -7.81 & 16.10 & 2313 & 23 & +492 & +221 & 19 \\
\hline NGC 1851 & & 051406.82 & -400247.0 & -1.22 & 0.02 & -8.33 & 15.47 & 3068 & 48 & +6 & +3 & 7 \\
\hline NGC 1904 & M 79 & 052411.31 & -243129.3 & -1.57 & 0.01 & -7.86 & 15.59 & 2356 & 10 & +11 & -2 & 10 \\
\hline NGC 2298 & & 064859.37 & -360020.0 & -1.85 & 0.14 & -6.30 & 15.59 & 999 & 4 & +2 & -1 & 68 \\
\hline NGC 2808 & & 091205.43 & -645153.9 & -1.15 & 0.22 & -9.39 & 15.59 & 4023 & 18 & -156 & +179 & 18 \\
\hline E 3 & & 092055.81 & $-77 \quad 1657.9$ & -0.80 & 0.30 & -2.77 & 14.12 & 380 & 0 & +62 & -386 & 82 \\
\hline NGC 3201 & & 101736.35 & -462441.1 & -1.58 & 0.23 & -7.46 & 14.21 & 2224 & 86 & +188 & -362 & 26 \\
\hline NGC 4147 & & $12 \quad 1006.34$ & +183232.4 & -1.83 & 0.02 & -6.16 & 16.48 & 1209 & 15 & -106 & +70 & 62 \\
\hline NGC 4372 & & 122550.56 & -723916.3 & -2.09 & 0.39 & -7.77 & 15.01 & 450 & 0 & +23 & +17 & 42 \\
\hline NGC 4590 & M 68 & 123928.00 & -264435.9 & -2.06 & 0.05 & -7.35 & 15.19 & 1202 & 42 & 0 & -1 & 20 \\
\hline NGC 4833 & & 125935.26 & -705230.2 & -1.80 & 0.32 & -8.16 & 15.07 & 1394 & 20 & +103 & -395 & 24 \\
\hline NGC 5024 & M 53 & 131254.91 & +181006.8 & -1.99 & 0.02 & -8.70 & 16.31 & 1686 & 63 & -5 & -3 & 14 \\
\hline NGC 5053 & & 131626.63 & +174200.9 & -2.29 & 0.04 & -6.72 & 16.19 & 1114 & 10 & +196 & -379 & 58 \\
\hline NGC 5139 & $\omega$ Cen & 132647.15 & -472849.5 & -1.62 & 0.12 & -10.29 & 13.97 & 8611 & 198 & -17 & -11 & 5 \\
\hline NGC 5272 & M 3 & 134211.54 & +282242.9 & -1.50 & 0.01 & -8.88 & 15.07 & 3461 & 241 & +16 & +9 & 4 \\
\hline NGC 5286 & & 134625.76 & -512215.0 & -1.67 & 0.24 & -8.61 & 15.95 & 2466 & 53 & -10 & +10 & 29 \\
\hline NGC 5634 & & 142936.95 & -055836.1 & -1.88 & 0.05 & -7.69 & 17.16 & 819 & 19 & -5 & -1 & 52 \\
\hline NGC 5694 & & 143936.87 & -263230.6 & -1.86 & 0.09 & -7.81 & 17.98 & 740 & 3 & +285 & -364 & 67 \\
\hline IC 4499 & & 150018.15 & -821255.6 & -1.60 & 0.23 & -7.33 & 17.09 & 1870 & 99 & +253 & +334 & 83 \\
\hline NGC 5824 & & 150359.06 & -330406.3 & -1.85 & 0.13 & -8.84 & 17.93 & 1924 & 26 & +7 & -1 & 54 \\
\hline NGC 5904 & M 5 & 151833.38 & +020452.0 & -1.27 & 0.03 & -8.81 & 14.46 & 3820 & 130 & +340 & -197 & 3 \\
\hline NGC 5927 & & 152800.96 & -504012.3 & -0.37 & 0.45 & -7.80 & 15.81 & 292 & 0 & +6 & +9 & 64 \\
\hline NGC 5986 & & $15 \quad 4603.27$ & $-3747 \quad 10.0$ & -1.58 & 0.28 & -8.44 & 15.96 & 1035 & 10 & -2 & 0 & 36 \\
\hline Pal 14 & AvdB & 161101.11 & +145731.0 & -1.52 & 0.04 & -4.73 & 19.47 & 465 & 0 & -55 & +2 & 116 \\
\hline NGC 6121 & M 4 & 162335.24 & -263133.3 & -1.20 & 0.36 & -7.20 & 12.83 & 5927 & 50 & +270 & -378 & 38 \\
\hline NGC 6101 & & 162547.01 & -721208.7 & -1.82 & 0.05 & -6.91 & 16.07 & 794 & 18 & +98 & -391 & 49 \\
\hline NGC 6205 & M 13 & 164141.50 & +362738.0 & -1.54 & 0.02 & -8.70 & 14.48 & 1269 & 9 & 0 & +1 & 2 \\
\hline NGC 6218 & M 12 & $1647 \quad 14.38$ & -015654.1 & -1.48 & 0.19 & -7.32 & 14.02 & 1324 & 2 & -2 & -2 & 23 \\
\hline NGC 6254 & M 10 & 165709.19 & -040601.5 & -1.52 & 0.28 & -7.48 & 14.08 & 4494 & 2 & +3 & -4 & 28 \\
\hline NGC 6341 & M 92 & $17 \quad 1707.73$ & +430803.4 & -2.28 & 0.02 & -8.20 & 14.64 & 1560 & 17 & +5 & -8 & 6 \\
\hline NGC 6366 & & 172744.45 & -050446.0 & -0.82 & 0.71 & -5.77 & 14.97 & 506 & 1 & +294 & -212 & 94 \\
\hline NGC 6656 & M 22 & 183623.28 & -235428.1 & -1.64 & 0.34 & -8.50 & 13.60 & 3438 & 31 & -42 & -15 & 17 \\
\hline NGC 6712 & & 185304.66 & -084208.4 & -1.01 & 0.45 & -7.50 & 15.60 & 494 & 15 & +333 & +21 & 70 \\
\hline NGC 6752 & & 191053.43 & -595908.4 & -1.54 & 0.04 & -7.73 & 13.13 & 8703 & 0 & -69 & +97 & 8 \\
\hline NGC 6760 & & 191112.60 & +010141.6 & -0.52 & 0.77 & -7.86 & 16.74 & 207 & 0 & +328 & -13 & 90 \\
\hline NGC 6809 & M 55 & 193958.78 & -305750.4 & -1.81 & 0.08 & -7.55 & 13.87 & 1772 & 15 & -8 & -7 & 16 \\
\hline Terzan 8 & & 194143.94 & -335959.6 & -2.00 & 0.12 & -5.05 & 17.45 & 404 & 3 & +465 & +121 & 117 \\
\hline Pal 11 & & 194514.62 & -080027.7 & -0.39 & 0.35 & -6.86 & 16.66 & 689 & 0 & +4 & -1 & 92 \\
\hline NGC 6838 & M 71 & 195346.53 & +184642.7 & -0.73 & 0.25 & -5.60 & 13.79 & 1266 & 1 & -94 & +1 & 65 \\
\hline NGC 6934 & & 203411.02 & +072416.8 & -1.54 & 0.10 & -7.46 & 16.29 & 743 & 79 & -7 & +2 & 53 \\
\hline NGC 6981 & M 72 & 205327.64 & -123204.4 & -1.40 & 0.05 & -7.04 & 16.31 & 1807 & 44 & -4 & +9 & 57 \\
\hline NGC 7006 & & 210129.37 & +161115.1 & -1.63 & 0.05 & -7.68 & 18.24 & 1780 & 64 & +50 & -348 & 66 \\
\hline NGC 7078 & M 15 & 212958.43 & +121002.9 & -2.26 & 0.10 & -9.17 & 15.37 & 2292 & 164 & +356 & +648 & 11 \\
\hline NGC 7089 & M 2 & 213327.37 & -004920.0 & -1.62 & 0.06 & -9.02 & 15.49 & 1185 & 38 & +7 & +84 & 9 \\
\hline NGC 7099 & M 30 & 214022.05 & -231043.7 & -2.12 & 0.03 & -7.43 & 14.62 & 616 & 7 & +32 & -13 & 15 \\
\hline NGC 7492 & & $2308 \quad 26.47$ & -153639.4 & -1.51 & 0.00 & -5.77 & 17.06 & 350 & 3 & +337 & +2 & 75 \\
\hline
\end{tabular}

\subsection{Cluster selection}

Initially, we prioritized GCs in the Harris $(1996,2010)$ catalogue based on their global properties, according to general criteria of scientific utility that should also make these GCs particularly suitable for study with the first Gaia data releases: (i) low reddening, and thus also low differential reddening, to make the CMD sequences easier to interpret; (ii) high total luminosity, to have better number statistics for model comparisons; (iii) low apparent distance modulus, to get better photometry at any given absolute stellar magnitude. By combining these three criteria, interstellar reddening is penalized twice because it makes the colours harder to interpret and extinction makes the stars appear fainter and more difficult to measure with precision. The number of RR Lyrae stars was also considered as an additional crite- 

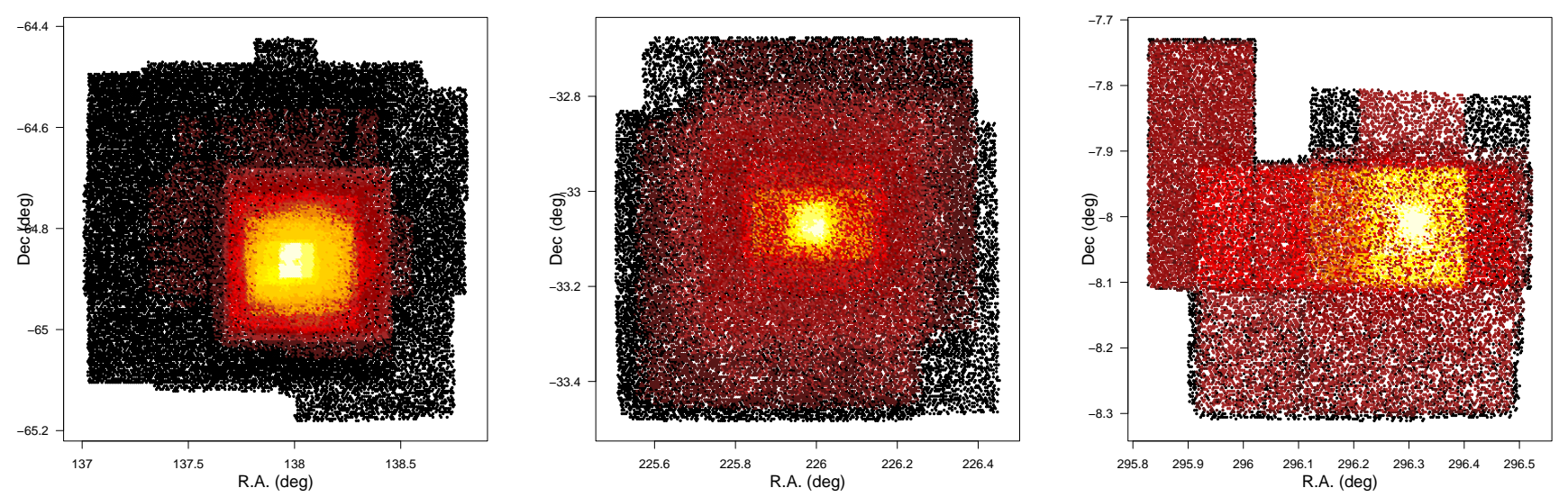

Figure 1. Examples of binned maps, with bins colored according to the maximum number of exposures (black lowest and yellow highest, varying as detailed below). Left panel: NGC 2808, one of the most covered GCs, with almost 4000 images used in the central regions (see Table 2). Center panel: NGC 5824, with almost 2000 images in the central regions and a more uniform coverage. Right panel: Palomar 11, with its peculiar image arrangement and $\simeq 650$ images in the center.

rion, but we found that it did not change the cluster ranking significantly.

Given the above prioritization, the GC choice was then finalized based on the availability of sufficient images obtained under photometric observing conditions ${ }^{2}$, the essential criterion being the possibility of deriving an accurate absolute calibration (see Section 3.1 for more details) in all five of the $U B V R I$ photometric bandpasses. The availability of $U$-band photometry is important because it is very sensitive to the CN molecular bands around $388 \mathrm{~nm}$, especially in red giants, which makes it a good indicator of multiple populations in GCs (Sbordone et al. 2011). The availability of the $R$ bandpass is less essential because for normal stars its information is largely redundant with that from the $V$ and $I$ bandpasses. Nevertheless, in order to somewhat limit the scope of this paper, we include in our present sample only clusters with fundamentally calibrated photometry in all five bandpasses.

This leads to the regrettable exclusion of high-priority GCs like NGC 6723 (ranked \#12 out of 150) or NGC 6541 (\#13), because they lack the $R$ filter, but it allows the inclusion of lower-priority GCs like Terzan 8 and Pal 14. We anticipate there will be a future paper like this one that will provide catalogues for clusters currently lacking the photometric $R$ band - a few of them, indeed, will have calibrated $R$-band photometry by the time the next paper is ready, since we are continuing to accumulate data. The final list of

\footnotetext{
2 Our working definition of "photometric" observing conditions is somewhat vague, but in general it means during the course of the night the r.m.s. dispersion of the fitting residuals for the standard stars observed is $\lesssim 0.03 \mathrm{mag}$, and the derived extinction coefficients seem reasonable. In our experience the extinction coefficients for a $2000 \mathrm{~m}$ mountain are $\sim 0.48$ in $U, 0.25$ in $B, 0.14$ in $V, 0.105$ in $R$, and 0.06 in $I$. La Palma, in particular, occasionally has significantly larger extinction coefficients due to dust blown from the Sahara. The actual r.m.s. residual observed in each filter on each night is included as a component of the uncertainty associated with each resulting magnitude measurement, which is passed along to subsequent stages of the analysis
}

GCs considered here, sorted by right ascension, is reported in Table 2, along with relevant GC properties.

\subsection{Data pre-reduction}

The photometric catalogues of the 48 GCs presented here are obtained from 84106 individual CCD images (not including standard fields) nominally in the Johnson-Cousins passbands (see Figure 1 and Table 2), plus an additional 9166 images obtained in other filters; these latter do not contribute to the calibrated photometry of this paper, but there were included in the reductions for the information they can provide toward the completeness of the star catalogues and the precision of the astrometry. The CCD images were collected with a variety of telescopes and cameras, as summarized in Table 1; in all, data from 1327 nights divided among 390 observing runs are employed here. More details on the data sources and the full image credits are given in Appendix A.

In a small minority of cases, notably data from the Mosaic cameras on the CTIO and KPNO $4 \mathrm{~m}$ telescopes and from MegaCam on the CFHT 3.6m telescope, and occasionally data contributed by colleagues, pre-processing of the images had already been performed. In the remaining cases pre-processing was done employing the bias frames, flat-field frames, etc. which had also been provided by the archives. If such calibration images were not available, we have not attempted to use the science images. Data were reduced with in-house routines and IRAF $^{3}$ tasks (Tody 1986, 1993). All images were corrected for direct current (DC) bias offset, trimmed to the good imaging section, and corrected with a two-dimensional bias masterframe. When suitable long/short flat-field images were available (rare), twodimensional corrections for shutter-timing errors were also applied. For the most part dark-current correction was not

3 IRAF is distributed by the National Optical Astronomy Observatories, which are operated by the Association of Universities for Research in Astronomy, Inc., under cooperative agreement with the National Science Foundation. 
performed under the assumption that dark current can be treated as a component of the sky brightness, which does not interest us and is in any case removed in the photometric analysis. On the very rare occasions when the raw images had no overscan region, the master bias frames removed the mean bias level as well as any stable two-dimensional electronic pattern; to the extent that DC bias levels fluctuated during the night, this also can be considered a component of the instantaneous sky brightness, which has no consequence for our photometric results.

Whenever both high-signal dome and sky flat-field images were available, we used the dome pattern to remove small-scale sensitivity variations, and the sky pattern to remove large-scale variations, by constructing a master flat $F=D \times\langle S / D\rangle$, where $D$ is the master dome flat, $S$ is the master sky flat, and $\langle S \mid D\rangle$ is a median-smoothed ratio of the sky and dome masterflats with a smoothing scale of order a seeing disk. Finally, bad-pixel maps were derived from median stacks of large numbers of science images and were used to mask the science images before photometric processing, both for images preprocessed by us and for those that had been preprocessed before we acquired them.

\section{PHOTOMETRY}

Instrumental magnitudes were measured with the PSFfitting packages DAOPHOT, ALLSTAR, and ALLFRAME (Stetson 1987, 1992, 1994) following procedures initially described in Stetson (1987), and amplified in, e.g., Stetson (1994). Candidate stars were identified in the images, initial stellar brightness and sky brightness estimates were derived from synthetic-aperture photometry, model PSFs were derived from the brightest and most isolated of the unsaturated stars in each image, and an initial pass of PSF photometry was performed. After this, stars overlooked by the star-finding algorithm were added by hand, and improved PSFs were obtained from the original images after all stars except the PSF stars had been provisionally subtracted from the images. Almost always the PSF was considered to vary quadratically with position within the images except when the number of available bright, isolated stars was insufficient (sometimes the case for early, small-format CCDs), or the fitting residuals gave convincing evidence that a quadratically varying model was not needed. Corrections relating PSF magnitudes to synthetic-aperture magnitudes were derived from growth-curve analysis of the PSF stars in images from which all other stars had been subtracted.

Multiple images of the same science field were registered to a common shared geometric reference system employing third-order polynomials (10 parameters in each of $x$ and $y$ ), again excepting a few early, small-format images where the number of stars was small and the higher-order distortions negligible. Then final PSF magnitudes were measured with the ALLFRAME routine imposing a common star list consistently to all overlapping frames. The simultaneous exploitation of information from all available images ultimately allows for deeper and more precise photometry, with the deepest and best-seeing images providing better positional constraints on faint detections as well as self-consistent deblending in crowded regions.

\subsection{Photometric calibration}

The goal of our calibration procedure is to transform the measured instrumental magnitudes to a photometric system as close to that of Landolt (1992) as possible. This is done using venerable methodology dating back to the beginnings of photoelectric photometry that takes into account both color and extinction corrections (see Appendix B). Our approach also allows for some variation of photometric zeropoints (ZPs) across the face of each two-dimensional detector (see Section 3.2), determined independently for each observing night and CCD. The average magnitudes and standard errors for each star are then obtained by robust statistical methods, with the procedure described in detail in Appendix B.

The final photometric calibration for each of our science targets is then performed in steps. Initially, candidate secondary photometric standards (Stetson 2000) are selected from each cluster catalogue by an automatic filtering process followed by visual inspection to identify stars with wellformed images that are also well isolated from neighbors. These are provisionally calibrated on the basis of Arlo Landolt's standards (Landolt 1992); stars with multiple concordant observations are considered valid secondary photometric standards and their results are added to our library of standard-star indices. This process is repeated for every science target field for which we have multiple observations obtained under photometric observing conditions, particularly when they came from multiple observing runs. This applies to all suitably observed targets: stars in OCs and GCs, stars in and near dwarf galaxies, stars in the fields of supernova hosts, stars in Landolt fields that were not themselves included in Landolt (1992), and all other targets meeting the basic acceptance criteria. At present, a candidate is accepted as a secondary standard if it satisfies the following criteria: (i) having at least five observations obtained under photometric observing conditions; (ii) a computed standard error of the mean magnitude in a given passband of $<0.02$ mag. Only passbands satisfying both these conditions are accepted for any given star, and a star is retained only if at least two of the five target magnitudes satisfy the criteria; magnitudes in other passbands not meeting these criteria are set to null values. Additionally, any stars that show evidence of intrinsic variability with r.m.s. dispersion $\geq 0.05$ mag considering all passbands together are rejected.

All these secondary standards are then used to augment the primary standards in refitting the calibration equations described in Appendix B for all CCDs on all nights of all observing runs. This recomputation does not necessarily improve the absolute reference of our global average photometric system to that of Landolt (1992). It does, however, help to ensure that each individual calibration equation for each CCD on each night of each run has been referenced to the same photometric system, and random photometric calibration errors resulting from small-number statistics are greatly reduced. This is especially beneficial for nights and runs where only a few Landolt standards were observed over a limited range of airmasses. It also greatly enhances the consistency even of runs where significant numbers of Landolt standards were observed. But especially it allows us, for the first time, to exploit data for our highly desirable science targets from runs where no Landolt standards 

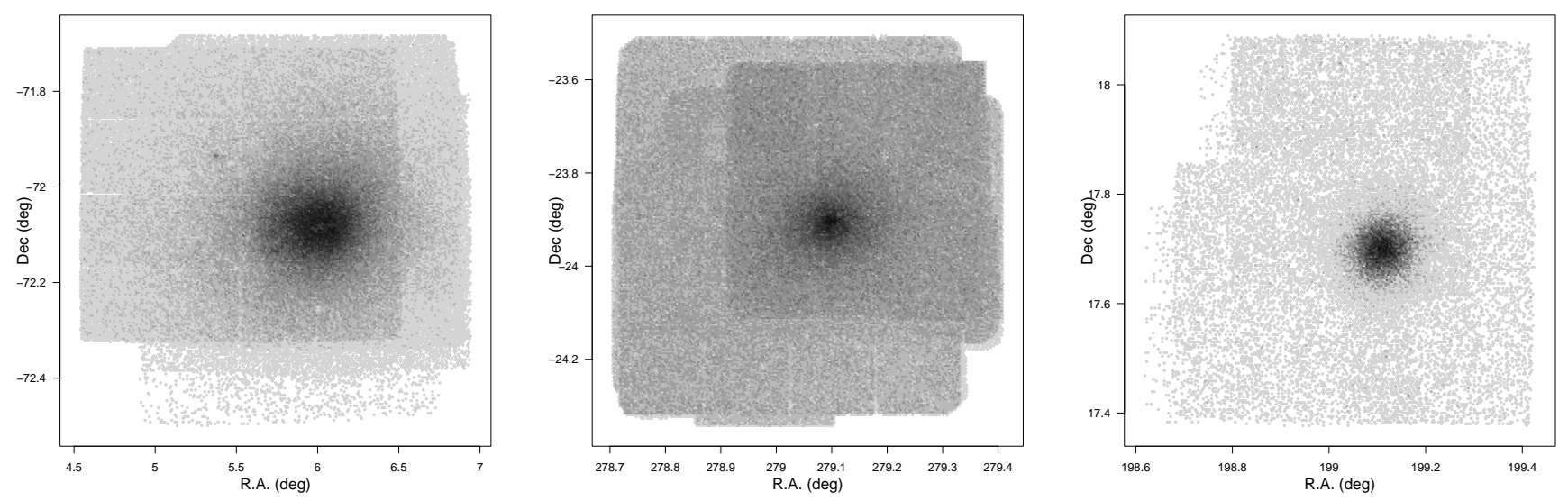

Figure 2. Examples of binned star density maps. Left panel: 47 Tucanae. The decrease in star counts caused by crowding is visible in the center, as well as the image footprints of mosaic cameras. The overdensity on the upper left of the cluster center is Bologna A (Bellazzini, Pancino \& Ferraro 2005). A hole caused by a very bright star is also apparent right below the GC central overdensity. Center panel: M 22, one of the most observed GCs in the sample. Right panel: the simpler case of NGC5053, whose photometry is based on fewer stars and CCD exposures.

were observed. To gauge the scale of the improvement, when Landolt (1973) photometry is adjusted for trivial ZP displacements and combined with the Landolt (1992) data, the resulting number of fundamental Landolt (1992) standards that meet our acceptance criteria $(n \geq 5, \sigma \leq 0.02$ in at least two of five filters) are 427, 451, 456, 301, and 295 stars in $U, B, V, R$, and $I$, respectively. As of this writing, our current working set of standards-Landolt's merged with our own list of secondary standards - contains 64 356, 164435 , 180661,88139 , and 155241 stars in $U, B, V, R$, and $I$, respectively. If we count only stars with $n \geq 100$ in a given filter, Landolt's merged catalogue contains seven stars in $U B V$ and three stars in $R I$; our combined list of primary and secondary standards contains 597, 15 109, 22 895, 3487, and 11931 in $U, B, V, R$, and $I$, respectively. These numbers are constantly growing as we continue to incorporate additional data. Accordingly, the last significant digit in each measured magnitude can change with time, but there is no secular drift of our system as we constantly monitor and remove any discrepancy with the fundamental Landolt standards, and the claimed standard error that we associate with each measurement is a realistic description of the instantaneous state of our knowledge.

Once the transformation-equation coefficients for the various run/night/chip/filter combinations have all been redetermined on the basis of the augmented standard-star list, somewhat relaxed acceptance criteria are adopted in choosing the local standards to be used in calibrating each individual science field. The criteria for local standards are (i) having at least three observations obtained under photometric observing conditions; (ii) a computed standard error of the mean magnitude of $<0.04 \mathrm{mag}$; (iii) no evidence of variability with r.m.s. $\geq 0.05$ mag based on all available filters. At this point we are assuming that all the transformation constants except the individual ZPs of the various images are known with a precision vastly better than the uncertainties of our individual instrumental magnitudes. This final step is intended to remove any unanticipated systematic $\mathrm{ZP}$ errors due to, for instance, unmodeled departures from the temporal and directional constancy of the derived extinction coefficients, or inadequacies in the derived corrections from a relative PSF magnitude scale to an absolute aperture-defined magnitude scale for each image. The individual photometric $\mathrm{ZP}$ of each image is redetermined via a robust weighted averaging technique that iteratively reduces the weight of discrepant observations (see lecture $3 \mathrm{~b}$ by Stetson 1989) producing a ZP that is optimally consistent with the preponderance of available calibrators. Accepting all other parameters as given and redetermining the average photometric ZP for each individual CCD image at this point is a last step in ensuring that all observations are on a common photometric system: "common" with a tolerance that is tighter than the tolerance to which it is "correct." Stated differently, redetermining the zero-point of every image at this stage produces the tightest possible principal sequences in the CMD, but does not affect the absolute placement of those sequences within colour-magnitude space.

To give an idea of the numbers involved, as of this writing the 48 GCs discussed here have a total of 61514 local secondary standards. The ZPs of the 84106 individual CCD images ${ }^{4}$ have been redetermined from a median of 155 local standards each. Among these, the median standard deviation of the magnitude residual for an individual star is $0.009 \mathrm{mag}$ in $\mathrm{U}, 0.004 \mathrm{mag}$ in $\mathrm{B}, 0.003 \mathrm{mag}$ in $\mathrm{V}, 0.007 \mathrm{mag}$ in $\mathrm{R}$, and $0.005 \mathrm{mag}$ in I. Using this approach, the robust weighted average results in a relative ZP whose reliability is not quite as good as the uncertainty of a single magnitude measurement divided by the square root of 155 . In fact the median uncertainty in the derived photometric $\mathrm{ZP}$ of a typical individual image is independently estimated ex post facto to be $\simeq 0.0015 \mathrm{mag}$ considering the r.m.s. repeatability of measurements in all filters and for all targets. The uncertainty contributed to the average calibrated magnitudes for a given star by the calibration itself is therefore of the order

4 We reduce and analyze each CCD separately even in case of mosaic cameras (Section B). For example, with WFI each exposure produces 8 individual CCD images. 

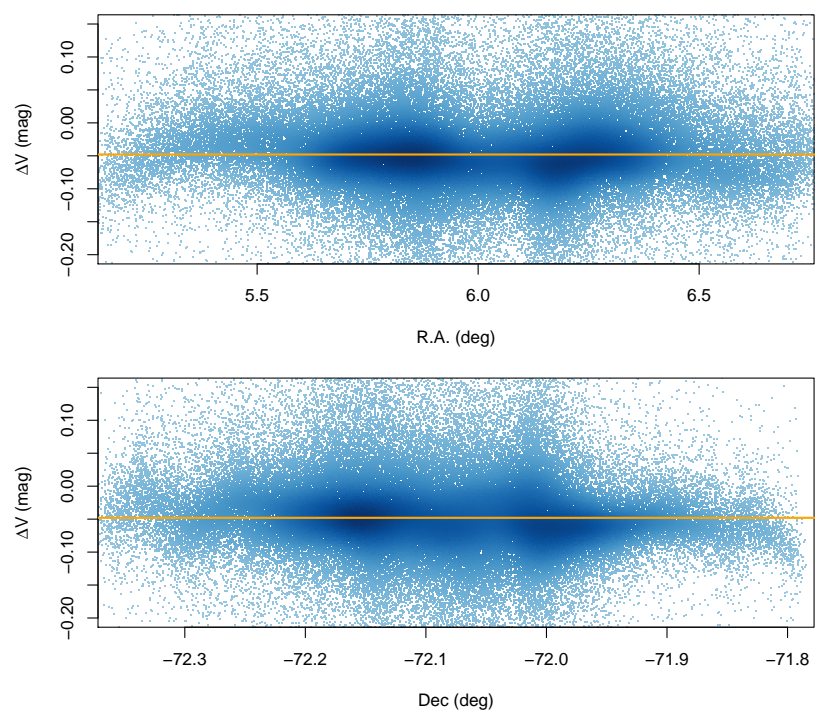

Figure 3. Example of $\mathrm{ZP}$ variations in wide field imagers. Our photometry of 47 Tucanae is compared with that of Kaluzny et al. (1998) and plotted as a function of RA (top panel) and Dec (bottom panel). The vertical stripes correspond to the overlap of different exposures in the Kaluzny et al. (1998) photometry. Part of the wavy patterns, especially at the borders, are caused by light concentration (see Section 3.2) in one or both catalogues.

of this number divided by the square root of the number of images where that star was measured in that bandpass. The actual limiting precision for a given average magnitude for a given star is therefore normally dominated by readout noise, photon statistics, and crowding errors in the individual images. We recall that errors in the instantaneous atmospheric extinction were removed by the redetermination of the ZPs of individual exposures.

\subsection{Position-dependent photometric errors}

In all cases the present photometry includes wide- (mosaic) as well as narrow-field (single-CCD) images (Table 1). The final combined areal coverage of our calibrated photometry ranges from a maximum extent as small as $35 \times 35$ arcmin for NGC 6101 to as much as $80 \times 80$ arcmin for NGC 288 (see other examples in Figure 2). In the crowded center of each cluster in our catalogue, where many images from many different instruments overlap (see Figure 2), any positiondependent photometric errors that remain after the minor spatial corrections that we have applied should be small, and should be further beaten down as the square root of the number of runs, nights, and dither offsets. They will certainly be less significant than systematic radial gradients in photometric bias produced by crowding in the cluster centers; crowding errors that affect the measured magnitudes should have a smaller effect on the colors that are derived from them, but users of these data should still be aware of possible small systematic errors in the colors of stars in the crowded cluster centers.

The outermost regions of each field can suffer in principle from minor large-scale ZP variations, aggravated by the fact that these regions typically are contained in the smallest number of CCD images obtained during the smallest num-

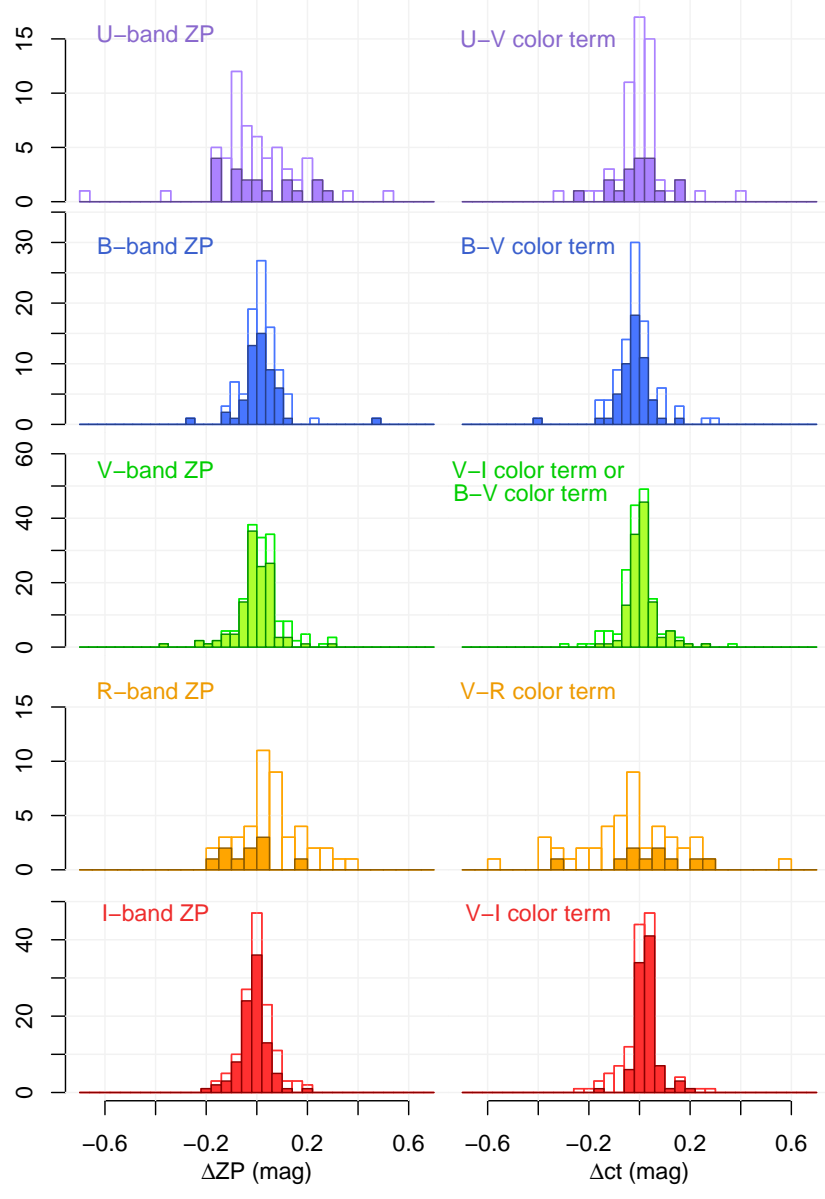

Figure 4. Results of the literature comparisons (see Section 3.3 and Table 3). Each row refers to a different photometric passband, as indicated. The left panels report histograms of the magnitude ZPs differences of the literature comparison, the right panels the corresponding color-term differences. Empty histograms include the fitted synthetic magnitudes by Pickles \& Depagne (2010), filled histograms report only the observed CCD measurements and photoelectric sequences.

ber of independent observing runs. An example is given in Figure 3, where our photometry of 47 Tuc is compared with that by Kaluzny et al. (1998), who report CCD linearity effects and calibration problems for fainter stars. The observed $V$ magnitude differences in the external regions can reach $\simeq 0.07-0.08 \mathrm{mag}$. If we humbly assign half of the difference to each study, we can place a generous upper limit to the maximum ZP variations of our photometry of $\lesssim 0.04 \mathrm{mag}$ in the external regions $(\gtrsim 0.5 \mathrm{deg}$ from the center). In fact, we believe that our careful calibration procedures produce results that are considerably better than this. Fortunately, any systematic photometric errors in the outer parts of each field affect primarily stars unassociated with the cluster.

\subsection{Literature comparisons}

We compared our photometric catalogues with the literature. Whenever possible, we preferred published photometry performed with techniques suited for crowded fields (viz., 
Table 3. Summary of literature comparisons (see also Figure 4 and Section 3.3). The available passbands are indicated, along with the number of stars and GCs in common with our catalogue.

\begin{tabular}{|c|c|c|c|c|c|c|c|}
\hline Reference & Bands & $\mathrm{n}_{\star}$ & GCs & Reference & Bands & $\mathrm{n}_{\star}$ & GCs \\
\hline Alvarado et al. $(1995)^{a}$ & $U B V R I$ & 19 & $2 G C s$ & Melbourne et al. (2000) & $B V I$ & 3237 & NGC 4833 \\
\hline Alves, Bond \& Onken (2001) & $B V$ & 5104 & NGC 5986 & McLaughlin et al. (2006) & $U V$ & 3757 & NGC 104 \\
\hline Anderson et al. (2008) & $V I$ & 358120 & 36 GCs & Mochejska et al. (2002) & $U B V$ & 11841 & NGC 6121 \\
\hline Bellazzini, Ferraro \& Ibata (2002) & $V I$ & 1634 & NGC 5634 & Monaco et al. (2004) & $B V I$ & 136753 & NGC 6656 \\
\hline Bellini et al. (2009) & $U B V R I$ & 339783 & NGC 5139 & Nardiello et al. (2015) & $U B V I$ & 3939 & NGC 6121 \\
\hline Bergbusch (1996) & $B V$ & 763 & NGC 7099 & Odewahn, Bryja \& Humphreys (1992) & $B V R$ & 133 & $2 G C s$ \\
\hline Buonanno et al. (1987) & $B V$ & 1331 & NGC 7492 & Ortolani \& Gratton (1990) & $B V$ & 2444 & $2 G C s$ \\
\hline Cohen et al. (2011) & $B V$ & 9683 & NGC 6101 & Pickles \& Depagne $(2010)^{b}$ & UBVRI & 4794 & $41 G C s$ \\
\hline Cote et al. (1995) & $B V$ & 315 & NGC 3201 & Pollard et al. (2005) & $B V I$ & 19811 & NGC 6254 \\
\hline de la Fuente Marcos et al. (2015) & $V I$ & 1061 & E 3 & Rees (1993) & $B V$ & 480 & NGC 5904 \\
\hline Ferraro et al. (1990) & $B V$ & 5420 & NGC 2808 & Rey et al. (2001) & $B V$ & 3949 & $2 G C s$ \\
\hline Ferraro et al. (1997) & $B V I$ & 17877 & NGC 5272 & Rey et al. (2004) & $B V$ & 76113 & NGC 5139 \\
\hline Feuillet, Paust \& Chaboyer (2014) & $B V I$ & 39169 & NGC 7078 & Rosenberg et al. (2000a,b) & $V I$ & 193788 & 29 GCs \\
\hline Geffert \& Maintz (2000) & $B V$ & 4030 & NGC 6838 & Saha et al. (2005) & $B V R I$ & 1617 & Pal 14 \\
\hline Guarnieri, Bragaglia \& Fusi-Pecci (1993) & $B V$ & 3371 & NGC 6205 & Samus et al. (1995) & $B V R I$ & 1368 & NGC 5286 \\
\hline Holland \& Harris (1992) & $B V$ & 320 & Pal 14 & Sarajedini \& Milone (1995) & $B V I$ & 776 & NGC 5053 \\
\hline Hilker (2006) & $B V$ & 141 & Pal 14 & Sariya, Yadav \& Bellini (2012) & $U B V I$ & 12435 & NGC 6809 \\
\hline Kaluzny et al. (1998) & $V I$ & 86523 & NGC 104 & Sariya \& Yadav (2015) & $B V I$ & 2326 & NGC 6366 \\
\hline Kravtsov et al. (1997) & $U B V$ & 2410 & NGC 1904 & Sollima et al. (2005) & $B V I$ & 94205 & NGC 5139 \\
\hline Kravtsov et al. (2009) & $U B V I$ & 12025 & NGC 3201 & Thomson et al. (2012) & $U B V I$ & 5852 & NGC 6752 \\
\hline Kravtsov et al. (2010) & $U B V I$ & 5124 & NGC 1261 & Walker (1994) & $B V I$ & 525 & NGC 4590 \\
\hline Kravtsov et al. (2014) & $U B V I$ & 16339 & NGC 6752 & Walker (1998) & $B V I$ & 4043 & NGC 1851 \\
\hline Lee \& Carney (1999) & $B V$ & 17366 & NGC 7089 & Wang et al. (2000) & $B V R I$ & 98 & NGC 4147 \\
\hline Lewis et al. (2006) & $V I$ & 5559 & Pal 11 & van Leeuwen et al. (2000) & $B V$ & 8237 & NGC 5139 \\
\hline Libralato et al. (2014) & $B V R$ & 55156 & $2 G C s$ & White $(1970)^{a}$ & $U B V$ & 43 & $5 G C s$ \\
\hline Lynga (1996) & $B V R$ & 2886 & NGC 5139 & Zaritsky et al. (2002) & $U B V I$ & 2267 & NGC 104 \\
\hline Marconi et al. (2001) & $B V I$ & 7921 & NGC 6101 & & & & \\
\hline
\end{tabular}

${ }^{a}$ Photoelectric sequences. ${ }^{b}$ Synthetic fitted magnitudes.

PSF photometry). In spite of the several hundreds of papers devoted to GC photometry in the literature, we found that only a limited number of studies actually published electronic catalogues. Moreover, some of the first CCD studies of the 1970's and 1980's published lists of measured magnitudes but did not publish any coordinates.

After some initial tests, we also excluded HST photometry based on WFPC2 (e.g., Piotto et al. 2002) because of the tiny field of view and too different spatial resolution. Among the HST studies, we chose the ACS survey of GCs (Sarajedini et al. 2007; Anderson et al. 2008) because of its wider field, but we had to cut out the GC centers to allow for a meaningful comparison with our ground-based data. We did not compare with the HST UV Legacy Survey (early data release, Piotto et al. 2015) because their $U$-band data (F336W) are not yet calibrated accurately enough to the Johnson system. There is also concern that the transformation equations between the HST filter and the ground-based $U$ system may depend on stellar temperature, reddening, metallicity, and gravity in some complex fashion, due to the inclusion of molecular bands, the Balmer convergence and jump, and the $\mathrm{H}$ and $\mathrm{K}$ lines of ionized calcium within the various filters' bandpasses. This is a potential topic for future, more detailed studies. Given the paucity of $U$ and $R$ measurements in the published literature, we also included in the comparisons the Pickles \& Depagne (2010) synthetic fitted magnitudes of Tycho 2 stars in the observed GC fields and some photoelectric sequences (White 1970; Alvarado et al. 1995). The list of literature studies finally considered is presented in Table 3. We did not include in the comparisons previously published versions of our own photometry.

We cross-correlated our catalogues with the literature with the CataPack software ${ }^{5}$. We then used a weighted linear model to fit the magnitude differences as a function of color, recognizing that our comparisons are biased towards giant stars, which generally have smaller measurement uncertainties than dwarfs, due to better photon statistics and smaller susceptibility to crowding effects. The results of all literature comparisons are summarized in Figure 4, where we plot histograms of the ZPs and the color terms of the magnitude differences.

The median ZP differences (our minus the literature) and the related semi-interquartile ranges are: $Z P_{U}=-$ $0.008 \pm 0.088, Z P_{B}=0.015 \pm 0.036, Z P_{V}=-0.003 \pm 0.035, Z P_{R}=-$ $0.026 \pm 0.058$, and $Z P_{I}=-0.009 \pm 0.028 \mathrm{mag}$. These figures compare well with the estimated accuracies provided in Section 3.1. The highly discrepant comparisons are just a few. Concerning the $U$ band, one should note that the filters used with various instruments can have significant differences from the standard Landolt $U$ transmission (with some surprising effects, see Momany et al. 2003), and that there are few literature sources to compare with. For the $R$ band there are even fewer literature studies.

The median values and semi-interquartile ranges of the corresponding color terms are: $C_{(U, U-V)}=-0.002 \pm 0.044$, $C_{(B, B-V)}=-0.015 \pm 0.027, \quad C_{(V, B-V),(V, V-I)}=0.001 \pm 0.019$,

\footnotetext{
${ }^{5}$ http://davide2.bo.astro.it/ paolo/Main/CataPack.html
} 
Table 4. Column-by-column description of the final catalogue, described in details in Section 4. The table is published in its entirety electronically and at CDS. The most updated version of each catalogue will also be available at the CADC.

\begin{tabular}{|c|c|c|}
\hline Column & Units & Description \\
\hline Cluster & & GC name \\
\hline Star & & Star ID (unique for each GC) \\
\hline $\mathrm{X}$ & $(\mathrm{sec})$ & $\mathrm{X}$ coordinate in arcseconds \\
\hline $\mathrm{Y}$ & $(\mathrm{sec})$ & $\mathrm{Y}$ coordinate in arcseconds \\
\hline$U$ & (mag) & Johnson U magnitude \\
\hline$\sigma_{U}$ & (mag) & U magnitude error \\
\hline $\mathrm{n}_{U}$ & & Number of $U$ measurements \\
\hline$B$ & (mag) & Johnson B magnitude \\
\hline$\sigma_{B}$ & (mag) & B magnitude error \\
\hline $\mathrm{n}_{B}$ & & Number of B measurements \\
\hline$V$ & (mag) & Johnson V magnitude \\
\hline$\sigma_{V}$ & (mag) & V magnitude error \\
\hline $\mathrm{n}_{V}$ & & Number of $\mathrm{V}$ measurements \\
\hline$R$ & (mag) & Cousins R magnitude \\
\hline$\sigma_{R}$ & (mag) & $\mathrm{R}$ magnitude error \\
\hline $\mathrm{n}_{R}$ & & Number of $\mathrm{R}$ measurements \\
\hline I & (mag) & Cousins I magnitude \\
\hline$\sigma_{I}$ & (mag) & I magnitude error \\
\hline $\mathrm{n}_{I}$ & & Number of I measurements \\
\hline & & DAOPHOT's $\chi$ parameter \\
\hline sharp & & DAOPHOT's sharp parameter \\
\hline vary & & Welch-Stetson variability index \\
\hline weight & & Weight of variability index \\
\hline RA & (hh mm ss) & Right Ascension \\
\hline Dec & (dd mm ss) & Declination \\
\hline
\end{tabular}

$C_{(R, V-R)}=0.055 \pm 0.062, \quad$ and $\quad C_{(I, V-I)}=0.023 \pm 0.021$ mag. In a minority of cases we observe that the color terms change visibly for bluer stars at the turnoff or on the upper main sequence, or for the horizontal branch (HB) stars. We ascribe this to an incomplete representation of surface gravities in the standards used to calibrate some of the literature photometry. In very few cases a small but perceptible second-order term was apparent from the plots, but we did not attempt to quantify it. The most striking case of a second-order term was in the comparison with the Pal 11 photometry by Lewis et al. (2006); the data for that study were not obtained under photometric conditions and were calibrated by comparison with other photometric samples.

For one specific GC, NGC 6760, we found no dedicated literature study and the GC appears only in the Piotto et al. (2002) sample. In general, literature photometry covers much smaller areas than the results we present here (with few exceptions: $\omega$ Cen, 47 Tuc, M 22), and very few studies included more than two or three bands. Additionally, the sample sizes of the literature studies are one order of magnitude (sometimes two) smaller than ours, although several contain comparable numbers of stars (for example Monaco et al. 2004; Bellini et al. 2009). The majority of our sample GCs has no previous $U$ or $R$ photometry publicly available in the literature.

\section{THE CATALOGUE}

The final photometric catalogue for each GC was obtained by robust weighted averages of the calibrated magnitudes obtained from the individual images (Section 3.1), taking into account the formal uncertainties in the individual magnitudes based upon readout noise, photon statistics, the quality of the profile fits, and the image-to-image repeatability (see Stetson 1987, 1992, 1994, and Appendix B for more details).

The combined catalogue format is summarized in Table 4 and reports for each passband the final average magnitude, its standard error of the mean $(\sigma)$, and the number of images employed in that passband. Each star in the catalogue is uniquely identified by specifying both the GC name and the star ID. The $x$ and $y$ pixel coordinates in arcseconds relative to an arbitrary origin are also tabulated. The $y$-axis $(x=0)$ is a great circle of right ascension, and the $x$-axis $(y=0)$ is tangent to a parallel of declination at the origin. The $x$ axis increases east and $y$ increases north. These coordinates are accurately on the system of the Gaia DR1 (Section 4.3) and should be precise enough to allow unambiguous cross-identification with other ground-based studies: the uncertainty of each position is $\sim 1 \%$ of the seeing disk. Other relevant quantities listed in Table 4 are detailed in the following sections.

\subsection{Quality indicators}

The catalogues are based on images obtained with different instruments and under different observing conditions, and the images cover diverse areas on the sky. Thus, the quality of the provided measurements can differ significantly for similar stars at different locations in a cluster. Different parameters can be used to judge the quality of measurements for each star and - depending on the specific science goalto select the most useful ones. A detailed discussion about how to select the best star list using the provided parameters is given by Stetson \& Harris (1988); here we briefly summarize the parameters presented in our catalogue.

The most obvious parameters are $\sigma$, the standard error associated with each magnitude measurement (see Stetson \& Harris 1988, for more details) and $n$, the number of independent measurements used to determine it. In addition, unlike most published photometry, the catalogue presented here contains all five Johnson-Cousins bands. This allows for colour-colour selections, that can sometimes separate in a very efficient way giants from dwarfs, or GC stars from field stars, or normal stars from abnormal ones or non-stellar objects, or badly measured stars from well measured ones.

Two additional quality parameters, specific to the DAOPHOT/ALLSTAR/ALLFRAME codes, are $\chi$ and sharp, described in detail in Stetson \& Harris (1988) and illustrated in Figure 5. The $\chi$ parameter measures the observed pixel-to-pixel scatter in the profile fits compared to the expected scatter from readout noise and photon statistics. The sharp parameter is a measure of how much of the badly modeled light is concentrated in the central pixels compared to the surrounding ones. It takes positive values for apparently extended objects (like background galaxies, lumps in nebulosity, or haloes around bright objects) and negative values for more pointed objects (like defective pix- 


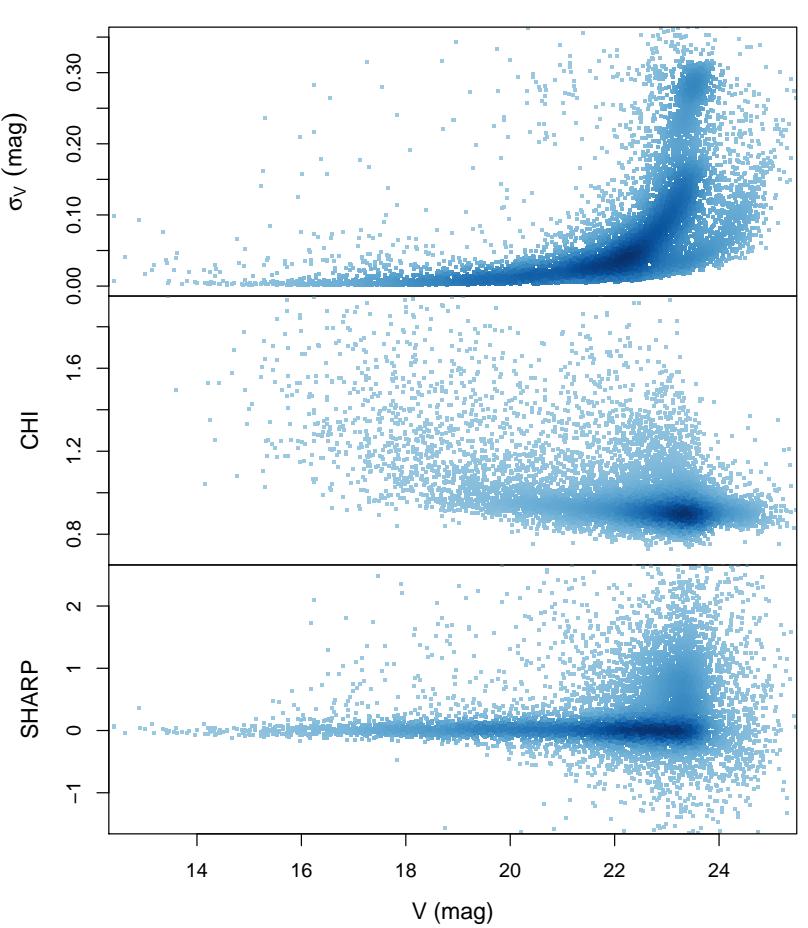

Figure 5. Example of image quality indicators for NGC 5694, plotted as a function of $V$ magnitude (see Section 4.1 for details). Top panel: the substructure in $\sigma_{V}$ at the faint magnitude end is caused by the varying depth of the used CCD images. Center panel: the bright stars deviate more from the typical PSF, but disturbed PSFs are apparent at all magnitudes. Bottom panel: at the faint magnitude end there are many cosmic ray hits and defective pixels (negative values) and diffuse objects (positive values).

els or cosmic ray hits), as shown in Figure 5. An example of CMD cleaning using these parameters can be found in Figure 6.

\subsection{Cluster centroids}

The GC centroids listed in Table 2 were recomputed in a consistent way with the procedure described in details by (Stetson, Hesser \& Smecker-Hane 1998). We suggest using these estimates, rather than literature centroids, in any procedure that requires a precise, self-consistent centroid determination, such as for example selection of stars in annuli to build star density profiles and the like (see Section 5.1).

The adopted procedure can be briefly summarized as follows. A virtual circular aperture is superimposed on the star catalogue down to a certain magnitude limit. The median $x$ and $y$ coordinates of the objects contained within the circular aperture are determined, and the aperture is shifted on the catalogue until the median coordinates of the objects contained in the circle coincide with the center of the circle. The exercise is repeated for aperture radii scaled by the fourth root of two, typically from 150 to 600 arcseconds. Two runs of this procedure are performed, one on the disks defined by the range $\left(0, r_{n}\right)$, and another on the annuli $\left(r_{n-1}, r_{n}\right)$. The overall range of the various centroid determinations is typically of order a few arcseconds, and the medians of the derived $x$ and $y$ centroids are adopted as the cluster photo-

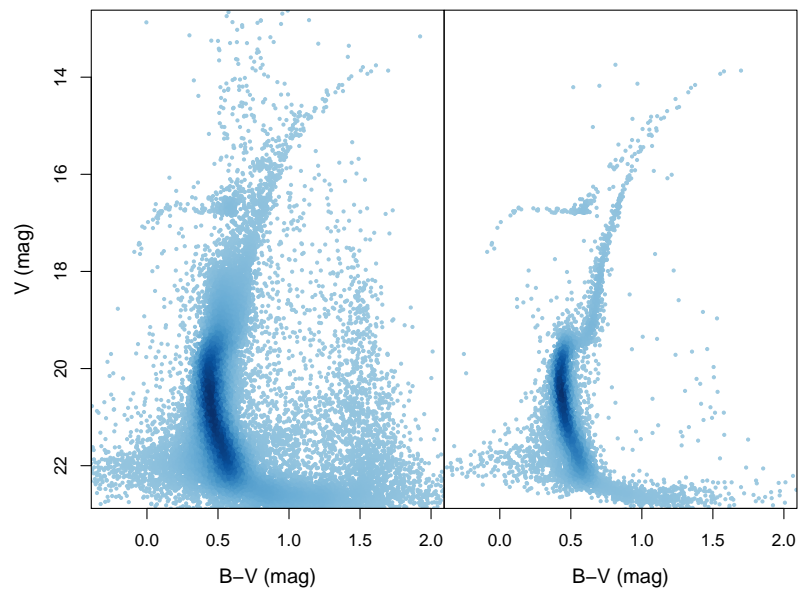

Figure 6. Example of CMD cleaning for NGC 1261. The left panel shows the $V, B-V$ CMD of the entire catalogue, the right panel an example selection, excluding stars within the core radius and outside $80 \%$ of the tidal radius from Harris (2010), and also excluding stars outside the range $-0.3<\operatorname{sharp}<0.3$, with $\chi>3$, or with $\sigma_{V}$ and $\sigma_{B}>0.2$ mag.

center, presuming that crowding errors, incompleteness, and other relevant effects will mostly be symmetric.

The samples enclosed by the various radii are not statistically independent, but the consistency observed even when smaller radii and various different magnitude limits are adopted indicates that the method is repeatable at the arcsecond level.

\subsection{Astrometric calibration on Gaia}

The relative astrometric calibration of star positions was performed using Gaia DR1 (the first data release, Gaia Collaboration et al. 2016a) as a reference.

The RA and Dec in Gaia DR1 are projected from the sphere onto an $(X, Y)$ plane by mapping position angle and angular distance relative to a chosen reference point on the sky to $\theta$ and $\rho$ in the plane. The angular coordinates are then transformed to $X$ and $Y$ by simple plane geometry. This differs from a gnomonic projection in that it preserves a constant scale in the radial direction to arbitrarily large angles, and the distortion in the circumferential direction is reduced. Specifically, if a gnomonic projection were applied to a field comprising half the sky, the scale of the projection would be an infinite number of planar units per arcsecond at a radial distance of 90 degrees from the tangent point. For the projection we use here, at a radial distance of 90 degrees from the reference point the scale of our planar $(X, Y)$ coordinates is one unit per arcsecond in the radial direction, and $\pi / 2$ units per arcsecond in the circumferential direction. The observed $(x, y)$ coordinates in the individual CCD images are then transformed to this master $(X, Y)$ catalogue using bivariate cubic polynomials with 20 plate constants. The transformed observed positions can then be converted to right ascension and declination in the Gaia DR1 system by reversing the mapping of the polar coordinates.

In a few cases, as illustrated e.g. in Figure 12 by Gaia Collaboration et al. (2016a), there were large gaps in the cat- 


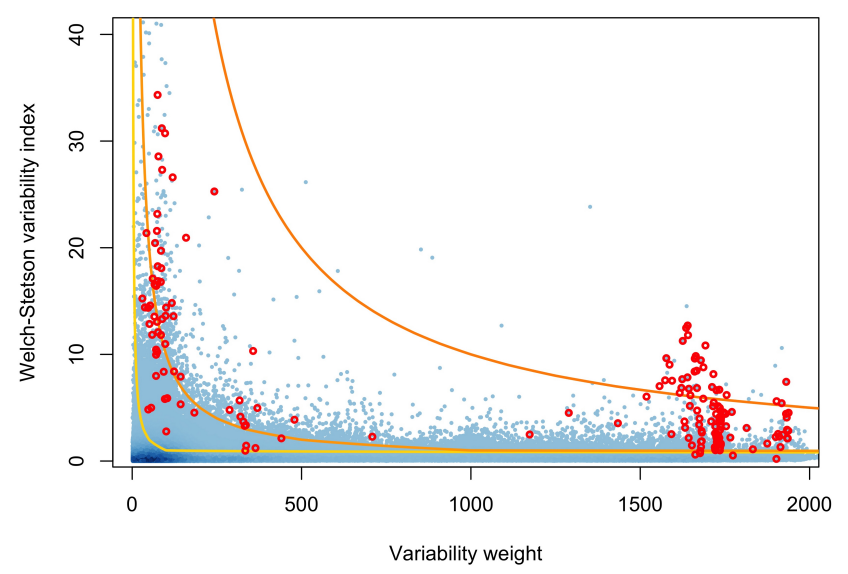

Figure 7. Behaviour of the variability indicators for the GC with the largest number of RR Lyrae variables: $\omega$ Centauri. The plot shows a zoom into the Welch-Stetson index versus variability weight plane of the entire catalogue (blue dots). The lines of evidence equal to 2 (gold), 3 (orange), and 4 (tomato) are overplotted for reference. Known RR Lyrae variables characterized by Braga et al. (2018) are overplotted in red: they mostly have variablity weight above $\simeq 30$ and evidence higher than $\simeq 2$.

alogues for some GCs as downloaded from Gaia DR1. We therefore also downloaded all available Digitized Sky Survey images of each field from the Canadian Astronomy Data Centre $^{6}$. The photographic coverage was generally selected to extend several arcminutes beyond the CCD coverage. Positions and magnitude indices for stars identified in these images were determined using the software described in Stetson (1979). The photographic results were mapped onto the Gaia catalogue as just described, and then the CCD data were mapped onto the merged Gaia/photographic catalogue.

\subsection{Variability indicators}

The photometric catalogue published here (Table 4) does not contain epoch data or light curves for variable stars; in a few cases these are published elsewhere, for example for NGC 4147, $\omega$ Cen, and M 4 (Stetson, Catelan \& Smith 2005; Braga et al. 2018). However, it contains useful information that can give an idea of which stars have a higher probability of being variable. The first indicator provided is the Welch-Stetson (W/S) variability index (Welch \& Stetson 1993), based on the correlation of the normalized residuals relative to the $U B V R I$ average magnitudes for observations obtained close in time. The second is the weight, equal to the number of residual pairs plus one-half the number of singleton residuals used to build the $\mathrm{W} / \mathrm{S}$ index. A useful derived parameter is the variability evidence, which we define as the logarithm of the $\mathrm{W} / \mathrm{S}$ index times its weight.

An example of the behaviour of the three indicators is shown in Figure 7. As can be seen, for low values of weight the $\mathrm{W} / \mathrm{S}$ parameter provides a large number of false positives and even relatively high values can be caused by image defects, crowding compounded with seeing variations, and other complications. Stars of the highest variability index

${ }^{6}$ http://www.cadc-ccda.hia-iha.nrc-cnrc.gc.ca/en/ and weight are the most likely to be real variables. Therefore, the "evidence" parameter can be an efficient way to pre-select and to rank stars for further examination: if stars are ranked in order of decreasing evidence and examined individually in that order, then by the time the investigator encounters (say) twenty false positives in a row it can be considered that the point of diminishing returns has been reached.

\section{RESULTS}

As mentioned above, the photometric catalogues provided here represent a significant improvement over previously published ground-based catalogues (Section 3.3) in terms of area, photometric bands, number of GCs homogeneously analyzed, number of stars per GC, and photometric quality. It will have a greater scientific impact once combined with proper motions from Gaia and abundances from spectroscopic surveys (Carretta et al. 2009a,b; Mészáros et al. 2015; Pancino et al. 2017), but we believe that it is already a valuable resource as it is. In the following sections, we show some selected new results, to illustrate the quality of the photometry and its potential for a rich scientific harvest. We have ideas for studies that we intend to perform, but we are making these data available to the community in the hopes that others will find uses that we ourselves have not yet considered.

\subsection{Cluster profiles}

Determining the structural properties of GCs is the first step in understanding their formation and evolution. The simplest observables for this purpose are the number-density and surface-brightness profiles, containing information on the spatial distribution of stars within each GC.

The only detailed compilation of surface brightness profiles for a large number of GCs is still the one by Trager, King \& Djorgovski (1995, containing 125 GCs), often computed combining surface brightness profiles extracted from mid-1980's CCD images and even older star counts on photographic plates. Care needs to be taken when using these profiles for mass-segregated clusters, for which the shapes of the number density and surface brightness profiles could differ significantly. Recently, surface brightness profiles for the innermost parts of 38 clusters have been measured with HST photometric data (Noyola \& Gebhardt 2006) and number density profiles for 26 Galactic globular clusters were presented by Miocchi et al. (2013). For some GCs, additional number-density and/or surface-brightness profiles have been calculated individually from high-quality photometry, for example: NGC 2419 (Bellazzini 2007); NGC 6388 (Lanzoni et al. 2007); $\omega$ Cen (Noyola, Gebhardt \& Bergmann 2008); and M92 (Di Cecco et al. 2013), among others. However, the only compilations of homogeneously computed profiles for a large number of globular clusters are the three studies mentioned above.

Here we consider M 5, one of the GCs in our sample with the most data and for which the images in all bands cover a similar extent, as an example of how the current version of our photometry database - of which we present the first 48 GCs here - could be used to compute high-quality 

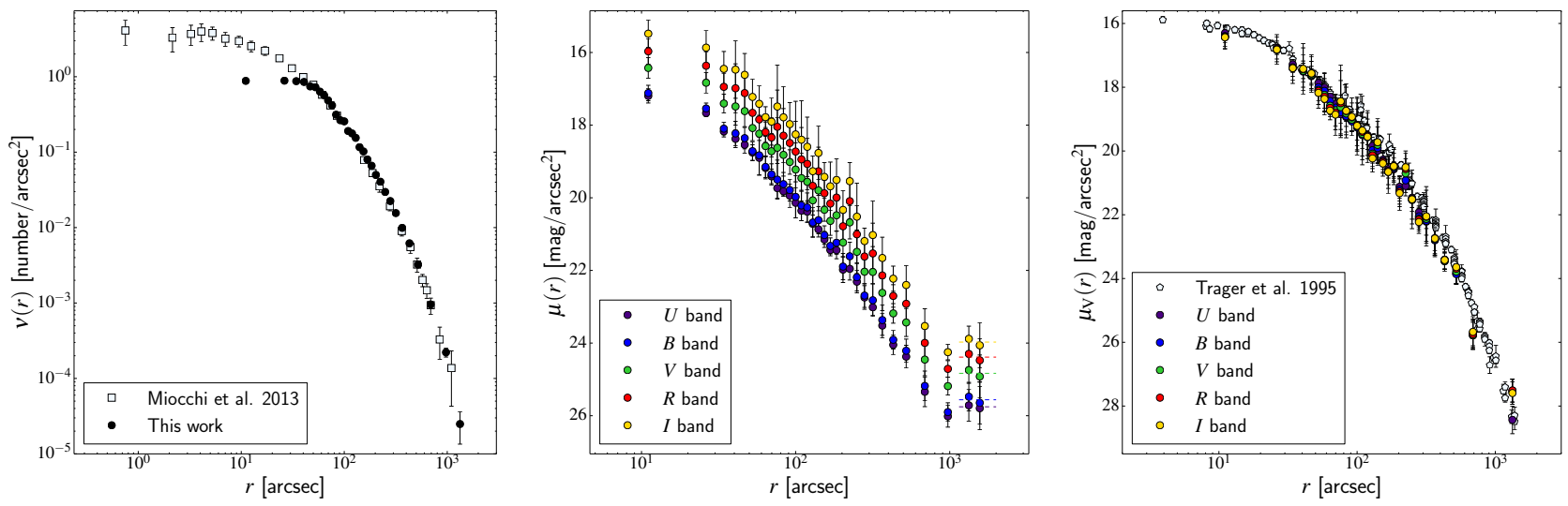

Figure 8. Example of number density and surface brightness profiles for the case of M 5. Left panel: background-subtracted number density profile $v$, as a function of the projected distance from the center, $r$ (black circles) compared to the profile by Miocchi et al. (2013, white squares). Center panel: surface brightness profile $\mu$ as a function of $r$. Violet, blue, green, red, and yellow circles represent the profiles calculated in the $U, B, V, R$, and $I$ bands, respectively. The dashed lines indicate the background level estimated for each band. Right panel: the surface brightness profiles, coloured as in the center panel, background-subtracted and opportunely shifted using the integrated color indices (see Section 5.1), and compared with the profile presented by Trager, King \& Djorgovski (1995, white pentagons).

surface-brightness and number-density profiles of GCs homogeneously. We consider stars brighter than $V=19$ mag and fainter than $V=12 \mathrm{mag}$, to ensure the catalogue is roughly complete at all radii and to avoid fluctuations due to very bright stars.

We computed the number-density profile by considering several concentric annuli of variable width, each containing 700 stars, to minimise the fluctuations in the profiles. The profiles are centered on the $\left(\mathrm{X}_{0}, \mathrm{Y}_{0}\right)$ centroids computed as described in Section 4.2 and listed in Table 2. Each annulus was divided into eight subsectors, where the number density was computed as the number of stars divided by the subsector's area. For each annulus, the number density and its error were computed as the mean and standard deviation of its subsectors' densities. We estimate the background density by considering the outermost regions, and we subtract this from the density profile. The resulting backgroundsubtracted number density profile, $v(r)$, with $r$ equal to the mean distance from the center for a given annulus, is shown in Figure 8 (left panel) where it is compared with the profile provided by Miocchi et al. (2013), showing excellent agreement. The discrepancy in the innermost part is due to crowding effects in our catalogue, as previously discussed.

We also computed the surface-brightness profiles, $\mu(r)$, in each of the $U B V R I$ photometric bands, by summing the corresponding flux in each subsector and then proceeding in the same way as for the number density, including the background subtraction. In this case, we also took into account the uncertainties in the individual luminosities to compute the standard error for each point in the profiles. Figure 8 (center panel) shows the resulting surface-brightness profiles. As can be seen, the profiles in the different bands have very similar shapes, and they overlap within the errors (Figure 8 , right panel) when shifted to the $V$ band using the integrated color indices provided in the Harris (2010) catalogue: $U-V=0.89, B-V=0.72, V-R=0.45$, and $V-I=0.95 \mathrm{mag}$. Indeed, the same figure shows a comparison between our shifted surface-brightness profiles and the surface-brightness profile provided by Trager, King \& Djorgovski (1995), which is quite satisfactory.

The profiles computed in this section are only presented as a function of the distance from the centre, assuming that the GC is spherically symmetric. Even though this is a common assumption, often adopted to describe GCs (and in particular it was adopted in the literature profiles we compare ours to), in Section 5.2 we will show that this is not necessarily true. With the present catalogues, it will be possible to compute density and surface brightness profiles without assuming spherical symmetry, and we plan to do this in the future.

\subsection{Cluster shape}

For a long time, GCs were considered spherically symmetric systems, and the observational profiles are commonly reported as a function of the distance from the centre only. While this is reasonable for GCs that do not exhibit perceptible flattening, for many other GCs, including M 5 (Section 5.1), significant deviations from spherical symmetry were detected (Geyer, Hopp \& Nelles 1983; White \& Shawl 1987; Chen \& Chen 2010). Unfortunately, because of incomplete sampling of different regions of GCs, the few available estimates of the global ellipticity could differ significantly from one work to another, as in the case of White \& Shawl (1987) and Chen \& Chen (2010). A homogeneous study of a large number of well-covered GCs is still sorely missing.

The photometry presented here has the potential to provide the basis for such a study, so we used NGC 5904 (M 5) as a test case for a method to quantify the deviation of the GC morphology from spherical symmetry ${ }^{7}$, that we will apply to our entire catalogue in the near future. For this GC, White \& Shawl (1987) report an ellipticity equal to 0.14. We consider all the stars brighter than $V=21 \mathrm{mag}$ and we do not

7 The method is loosely based on those employed by Danilov \& Seleznev (1994) and Pancino et al. (2003). 

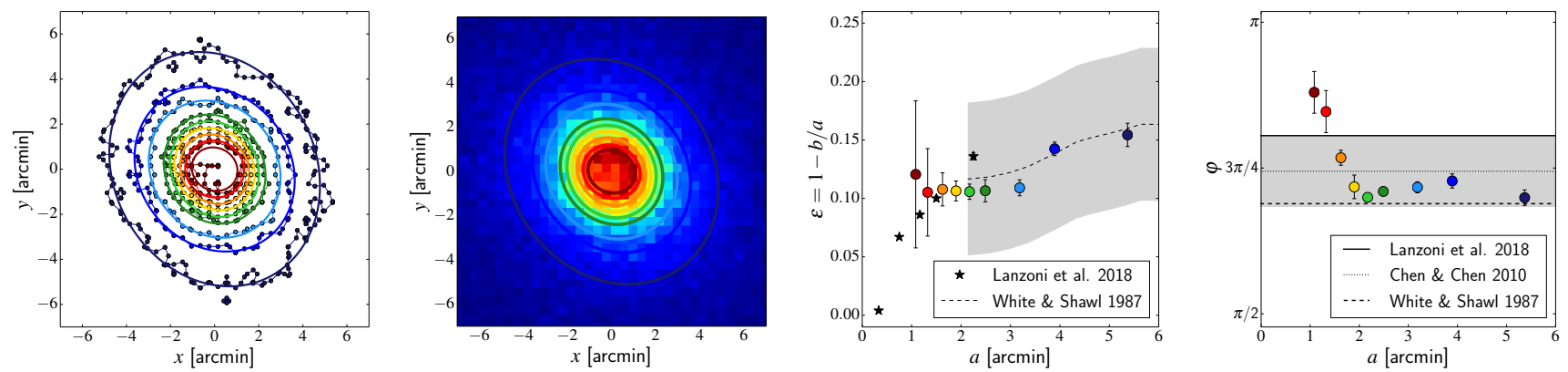

Figure 9. Morphology of NGC 5904 (M 5). In all panels, colours consistently refer to the selected levels of the projected number density, listed on Table 5. Left panel: Isodensity contours (dots and thin solid lines) and their corresponding best-fit ellipses (thick solid lines) for a particular choice of the grid (Section 5.2). Center-left panel: the same best-fit ellipses are overplotted on the density map of M 5, where each box corresponds to a cell of the chosen grid. Center-right panel: ellipticity profile $\varepsilon(a)$ as a function of the semi-major axis of the ellipses, coloured as in the preceding panels. The black stars report the ellipticity estimated by Lanzoni et al. (2018) and the dashed line the ones by White \& Shawl (1987), with their errors represented by the grey shaded area. Right panel: position angle $\varphi(a)$ as a function of the semi-major axis of the ellipses. The dashed, solid, and dotted lines correspond, respectively, to the values determined by White \& Shawl (1987), Lanzoni et al. (2018), and Chen \& Chen (2010), with the uncertainty on the last one shown as a grey shaded area.

correct for the field population, because it is reasonable to assume it to be uniform across the extent of the cluster.

We start by considering a grid covering the area occupied by NGC 5904, made of $0.4^{\prime} \times 0.4^{\prime}$ cells, defined in the $x$ and $y$ coordinates (Section 4$)$ and centered on the $\left(\mathrm{X}_{0}, \mathrm{Y}_{0}\right)$ centroid of NGC 5904 (see Table 2 and Section 4.2). The size of the cells is small enough to provide a good representation of the GC and large enough to avoid excessive fluctuations due to the discrete nature of the dataset. For each cell, identified by the position of its centre on the plane, we compute the number density and we interpolate it as a function of $x$ and $y^{8}$. We consider several contours for the interpolating function, corresponding to selected fractional values $f_{\text {peak }}$ of the peak value, in particular we use $f_{\text {peak }}=0.85,0.8,0.7,0.6$, $0.5,0.4,0.25,0.17$, and 0.08 . We then determine the parameters of the ellipse that provides the best fit for each of the contours thus obtained ${ }^{9}$ : the semi-major axis $a$, the semiminor axis $b$, the counterclockwise angle of rotation from the $x$-axis $\varphi$, and the position of the centre of the ellipse $\left(x_{\mathrm{e}}, y_{\mathrm{e}}\right)$.

We repeated this procedure several times, shifting the position of the grid: we considered 60 different positions for the grid, each time shifting it horizontally and vertically by $3^{\prime \prime}$. For each contour, the final values of the parameters and their errors are listed in Table 5, and correspond to the mean and standard deviation of the best-fit values obtained at each centring of the grid. This procedure allows us to prevent fluctuations in the number density due to the discrete nature of the sample from influencing the morphology determination.

The two left panels of Figure 9 show the results in graphical form, for one particular choice of the underlying grid. It can be seen that the innermost profile $\left(f_{\text {peak }}=0.85\right)$ is

\footnotetext{
8 We use the number density and not the surface brightness because the former is more stable: when evaluated on a grid, the surface brightness fluctuates a lot more and makes it difficult to obtain reliable contours.

9 To determine the ellipse best-fit parameters, we use the code by http://nicky.vanforeest.com/misc/fitEllipse/fitEllipse.html
}

Table 5. Best-fit parameters identifying the ellipses corresponding to the selected number density contours for M 5: semi-major axis $a$; semi-minor axis $b$; counterclockwise angle of rotation from the $x$-axis to the major axis of the ellipse $\varphi$ (note that the position angle recorded here also corresponds to the angle between the North and the semiminor axis of the ellipse, going towards East; therefore, the angle between the North and the semimajor axis of the ellipse can be found by simply subtracting $\pi / 2$ from the values of $\varphi$ ); ellipticity $\varepsilon=1-b / a$; position of the centre of the ellipse $\left(x_{\mathrm{e}}, y_{\mathrm{e}}\right)$; fraction of total stars contained within the corresponding contour $f_{\text {int }}$; and value of the density on the contour expressed as a fraction of its peak value $f_{\text {peak }}$.

\begin{tabular}{ccccccc}
\hline \hline $\begin{array}{c}a \\
\left({ }^{\prime}\right)\end{array}$ & $\begin{array}{c}b \\
\left({ }^{\prime}\right)\end{array}$ & $\begin{array}{c}\varphi \\
(\mathrm{rad})\end{array}$ & $\varepsilon$ & $\begin{array}{c}\left(x_{\mathrm{e}}, y_{\mathrm{e}}\right) \\
\left({ }^{\prime \prime},,^{\prime \prime}\right)\end{array}$ & $f_{\text {int }}$ & $f_{\text {peak }}$ \\
\hline $1.12 \pm 0.06$ & $0.94 \pm 0.05$ & $2.73 \pm 0.09$ & $0.16 \pm 0.06$ & $(6.0,6.3)$ & 0.10 & 0.85 \\
$1.33 \pm 0.03$ & $1.19 \pm 0.04$ & $2.64 \pm 0.11$ & $0.11 \pm 0.03$ & $(2.2,3.9)$ & 0.14 & 0.80 \\
$1.63 \pm 0.02$ & $1.45 \pm 0.01$ & $2.39 \pm 0.05$ & $0.11 \pm 0.01$ & $(-0.2,2.5)$ & 0.20 & 0.70 \\
$1.89 \pm 0.01$ & $1.70 \pm 0.01$ & $2.25 \pm 0.07$ & $0.10 \pm 0.01$ & $(-1.3,3.3)$ & 0.26 & 0.60 \\
$2.17 \pm 0.01$ & $1.94 \pm 0.01$ & $2.20 \pm 0.02$ & $0.10 \pm 0.01$ & $(-0.5,3.1)$ & 0.32 & 0.50 \\
$2.49 \pm 0.02$ & $2.22 \pm 0.02$ & $2.24 \pm 0.02$ & $0.11 \pm 0.01$ & $(0.6,2.5)$ & 0.37 & 0.40 \\
$3.18 \pm 0.02$ & $2.84 \pm 0.02$ & $2.25 \pm 0.03$ & $0.11 \pm 0.01$ & $(0.8,2.2)$ & 0.49 & 0.25 \\
$3.89 \pm 0.02$ & $3.34 \pm 0.01$ & $2.29 \pm 0.04$ & $0.14 \pm 0.01$ & $(1.0,1.6)$ & 0.57 & 0.17 \\
$5.37 \pm 0.06$ & $4.54 \pm 0.02$ & $2.20 \pm 0.04$ & $0.15 \pm 0.01$ & $(-1.1,2.3)$ & 0.70 & 0.08 \\
\hline \hline
\end{tabular}

irregular, and has the shape of a horseshoe, probably caused by stochastic effects and possibly by incompleteness due to stellar crowding in the GC center; the best-fit ellipse in this case needs to be taken with caution.

For each isopleth, we compute the ellipticity as $\varepsilon=$ $1-b / a$. We show the values of $\varepsilon$ as a function of the semimajor axis $a$ in the center-right panel of Figure 9, where we also compare our results with those of Lanzoni et al. (2018) and White \& Shawl (1987). We note how the contours corresponding to smaller densities appear to be more flattened, in agreement with what is found in the studies of NGC 5904 already cited: a similar behaviour was also observed in other globular clusters (Geyer, Hopp \& Nelles 1983; White \& Shawl 1987). The improvement in the number and the quality of the measurements in the ellipticity profile is evident, and the agreement with the profile by White \& Shawl (1987) in the external parts of NGC 5904 is remark- 

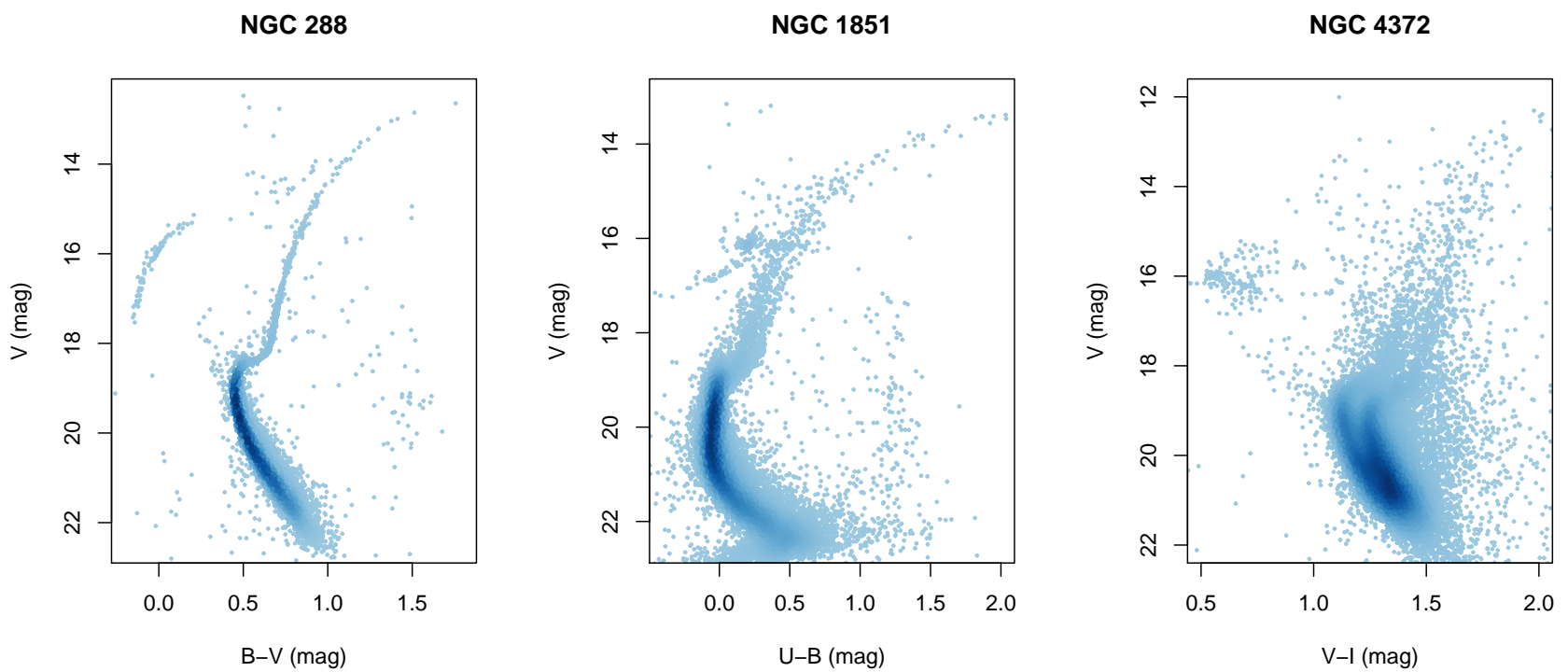

Figure 10. Examples of CMDs, cleaned with procedures such as the ones described in Section 4.1. The adopted selections sacrifice photometric depth to enhance the CMD details for stars brighter than V 20.5 mag (the approximate Gaia limit). Left panel: the welldefined CMD of NGC 288, with its beautiful binary and blue straggler stars sequences. Center panel: the complexity and fine structure of the RGB of NGC 1851 seen in $U-B$ colour. Right panel: the striking effect of differential reddening on the CMD of NGC 4372 , which appears bifurcated, with a redder turnoff dominated by stars north of the GC center and a bluer one dominated by stars south of it.

able. Figure 9 (right panel) also illustrates the behaviour of $\varphi$, the position angle of the fitted ellipses, measured as the counterclockwise angle between the $x$-axis and the semimajor axis of the ellipse. A small change in $\varphi$ is observed in the innermost part of the profile, but the outermost appears to be consistent with a single orientation, corresponding to $\varphi \lesssim 3 \pi / 4$. The change in $\varphi$ in the innermost region of the cluster needs to be taken with care because of the completeness issues in the centre. A comparison with the global $\varphi$ estimates by White \& Shawl (1987), Lanzoni et al. (2018), and Chen \& Chen (2010) is also shown, and reasonable agreement is found. The centers of the ellipses $\left(x_{\mathrm{e}}, y_{\mathrm{e}}\right)$ obtained for the considered levels are always within some arcsec from each other and from $\left(\mathrm{X}_{0}, \mathrm{Y}_{0}\right)$ : the largest distance from the GC centroid is found for the innermost ellipse, and is of $\simeq 8^{\prime \prime} .7$, possibly another manifestation of crowding issues in the GC central regions.

Often, the ellipticity of the contour containing half the mass of a star cluster is assumed to be a good global indication of its morphology (Kontizas et al. 1989). We therefore compute the fraction of the total number of stars that is enclosed in every contour, $f_{\text {int }}$ (Table 5 ), and by interpolating it as a function of $f_{\text {peak }}$ we determine $\varepsilon=0.11 \pm 0.01$ at $f_{\text {int }}=0.5$ for NGC 5904. Another possible estimate of the global ellipticity of the cluster can be calculated as the mean of the ellipticities for all the considered contours, which in the present case results to be $\varepsilon=0.12 \pm 0.02$, consistent with the value $(\varepsilon=0.14)$ calculated in a similar way by White $\&$ Shawl (1987).

The results presented here show very good agreement with the estimates available in the literature, but provide a much richer level of detail. The ellipticity profile computed by White \& Shawl (1987) extends farther out than the one calculated here, but it has significantly larger uncertainties.
This illustrates the impact that the photometry presented here can have in the study of GC morphology and ellipticity profiles. Combining these catalogues with Gaia data and with HST photometry (Anderson et al. 2008; Milone et al. 2017), it will be possible to extend the profiles to cover both the most external regions and the innermost crowded cores.

\subsection{Colour-magnitude diagrams}

The quality of the CMDs varies significantly depending on several factors, including: the depth and number of the collected images; the atmospheric conditions; the telescopes and detector quality; the sky coverage; galactic coordinates and hence both field-star contamination and the amount of reddening, especially differential reddening; and last but not least, the intrinsic properties of each GC. Consequently, some of the GCs can only be studied after filtering the data with techniques suited to the needs of the specific scientific investigation. In Section 4.1, we described the quality parameters that are included in the catalogue and that can be used to pre-select stars (see also Stetson \& Harris 1988, for more details). Additional external constraints can be extremely useful: for example a differential reddening correction can be computed following methods similar to that by Milone et al. (2012), or proper motions from Gaia or other sources can be used to select probable members.

To illustrate the variety in our photometric catalogue, we present in Figure 10 the CMDs of three GCs in different passbands. Our $V, B-V$ CMD of NGC 288 reveals in detail the beautiful binary sequence and blue-straggler plume that were originally shown by Bolte (1992, with ground-based photometry) and Bellazzini et al. (2002, with HST photometry). NGC 1851 is one of the so-called anomalous GCs that show an additional sub-population compared to the typical 

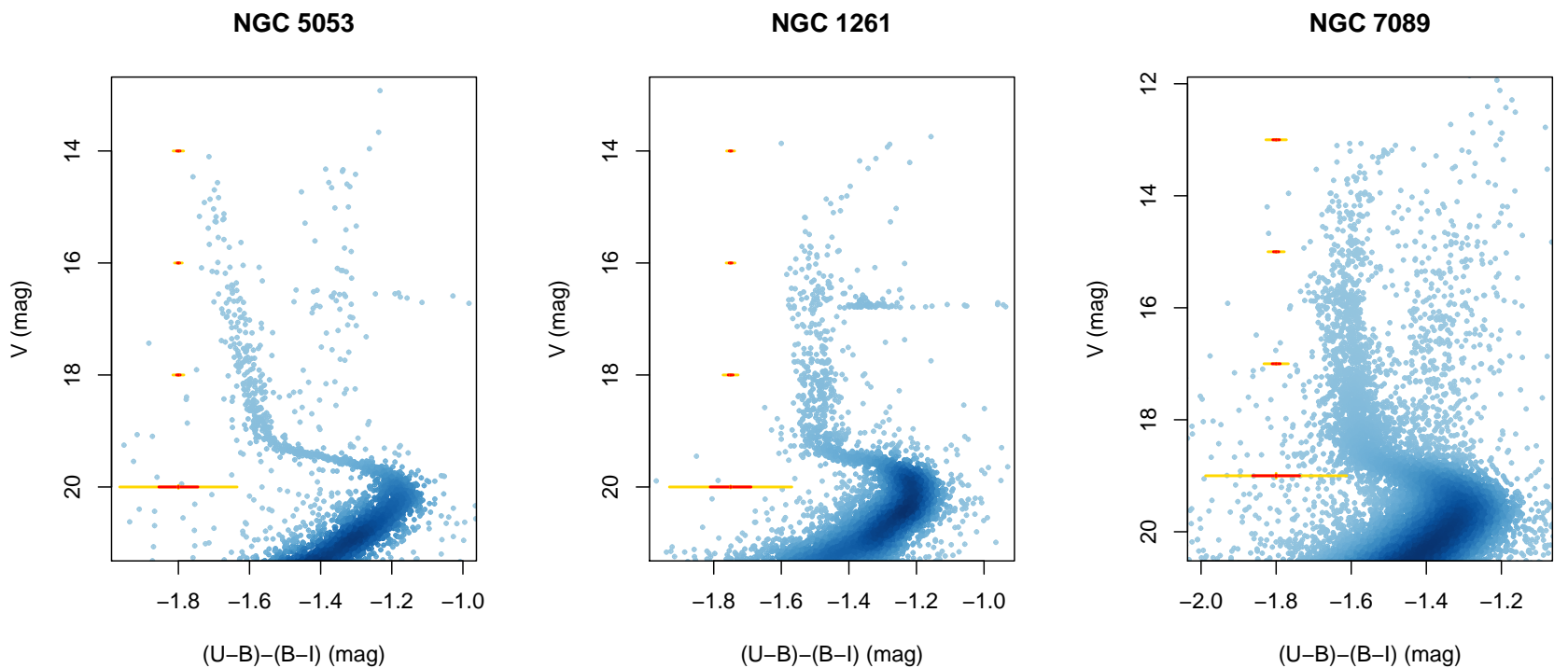

Figure 11. Examples of $V, C_{U B I}$ CMDs for three well-known GCs that were not included in the sample presented by Monelli et al. (2013), sorted by RGB width and complexity. Left panel: NGC 5053, one of the most metal-poor GCs displaying one of the thinnest RGBs in our sample, although still a few times wider that the typical photometric errors. Center panel: the beautiful substructure of the RGB of NGC 1261, a low-reddening GC. Right panel: the complex structure of another anomalous GC, NGC 7089 (M 2), containing a small fraction of s-rich and $\mathrm{C}+\mathrm{N}+\mathrm{O}$ enhanced stars, which are however not clearly visible in $C_{U B I}$. In all panels, median errorbars are displayed in gold $(3 \sigma)$ and red $(1 \sigma)$ at different magnitude levels.

GCs, in this case redder than the RGB and fainter than the subgiant branch (SGB), with enhanced $\mathrm{C}+\mathrm{N}+\mathrm{O}$ and sprocess element abundances (Lardo et al. 2012). Some other well-known GCs in this category are, for example, $\omega$ Cen (Lee et al. 1999; Pancino et al. 2000; Ferraro et al. 2004; Marino et al. 2012a), M 22 (Marino et al. 2009, 2012b), and M 2 (Lardo et al. 2013). The $V, U-B$ CMD that we present here shows the fine substructure of the RGB of NGC 1851 with its anomalous, additional red RGB that appears well separated from the remainder of the RGB populations. The main RGB in turn is widened (and possibly bimodal), a typical characteristic of all GCs, caused primarily by nitrogen variations (Monelli et al. 2013; Sbordone et al. 2011).

We also show in Figure 10 the peculiar effect that differential reddening has on the appearance of the $V, V-I$ CMD of NGC 4372. A sharp discontinuity in interstellar medium runs diagonally across the cluster's central regions, splitting stars into two well-separated sets of sequences (Gerashchenko \& Kadla 2004). This is especially visible in the turnoff region of our $V, V-I \mathrm{CMD}$, where a bluer turnoff is dominated by stars lying south of the GC center, and a redder one by stars north of it.

\subsubsection{Multiple populations}

A full statistical analysis of the photometric properties of MPs in GCs is clearly beyond the scope of the present paper. Nevertheless, we show the potential of our catalogue for separating and classifying MPs using the $C_{U B I}$ index defined by Monelli et al. (2013) — based on the index by Milone et al. (2013) - as $(U-B)-(B-I)$. In normal stars of spectral classes $\mathrm{G}$ and $\mathrm{K}$ (roughly speaking, $0.6 \lesssim B-V \lesssim 1.5 \mathrm{mag}$, or $1.5 \lesssim B-I \lesssim 3.7 \mathrm{mag})$ both $B-I$ and $U-B$ are strongly dependent on temperature, with a slope $d(U-B) /(B-I) \sim 1{ }^{10}$ The $(U-B)-(B-I)$ color difference, therefore, almost completely removes any temperature sensitivity, unmasking the smaller effects due to variations in carbon- and nitrogen-sensitive features - especially the strong CN band at $388 \mathrm{~nm}$ - and the effect of helium abundance on the strength of the Balmer convergence and jump. It is noteworthy that the reddening slope for stars of these colors is quite different: $E(U-B) / E(B-I) \sim 0.4$, so significant differential reddening reduces the effectiveness of the $C_{U B I}$ index.

In the study by Monelli et al. (2013), a variety of $C_{U B I}$ morphologies was illustrated, and we find the same variety in the present sample. Some GCs clearly show a split RGB in the $V, C_{U B I} \mathrm{CMD}$, with two well-separated branches: the two most striking examples in our sample are NGC 288 and NGC 6981. Some other GCs show (at least) three RGBs, like NGC 6205, NGC 1851, NGC 2808, $\omega$ Cen, or 47 Tuc. However in several cases the RGB is not clearly separated in distinct branches, and it rather shows a complex but not fully discrete morphology. For a few GCs namely E 3, NGC 2298, NGC 4833, NGC 5927, NGC 6760, NGC 6838, and NGC 7006 - differential reddening and/or field contamination blur the RGB and thus a dedicated treatment of these effects is needed before studying their MPs in details.

10 A plot of Landolt's mainly Population I standard stars, including both giants and dwarfs, is well represented by a line segment from $(B-I, U-B)=(1.5,0.1)$ to $(3.7,2.1)$. Metal-poor stars are shifted to smaller $U-B$ colors by their lower line blanketing, but their sequence remains nearly parallel to the Population I sequence in this temperature range. 
Terzan 8

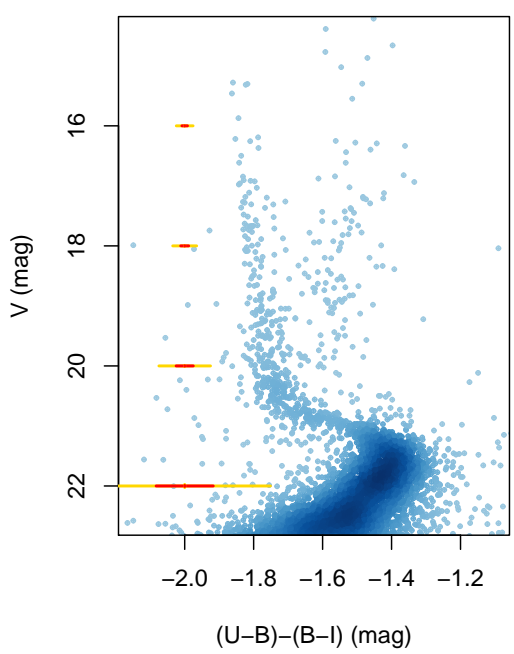

NGC 5694

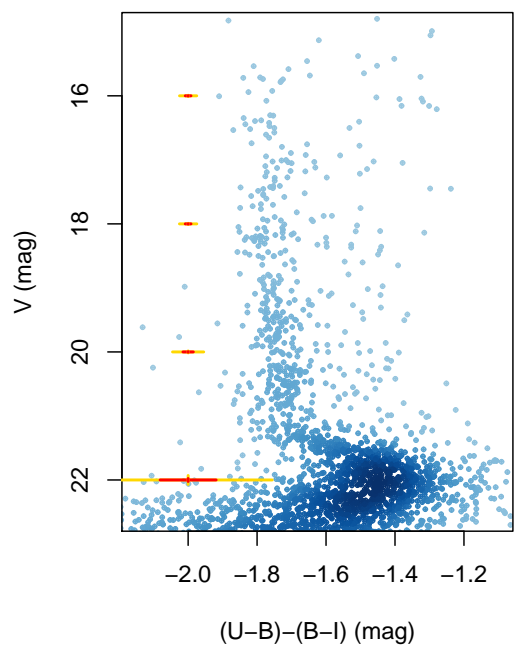

Palomar 14

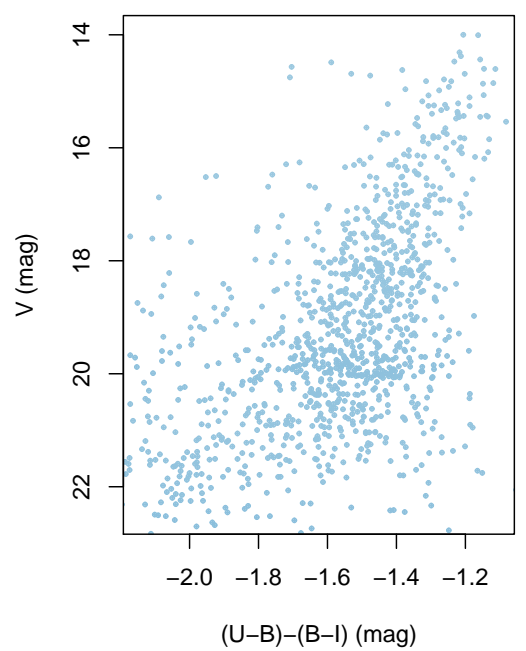

Figure 12. The $V, C_{U B I}$ CMDs of candidate single-population or mostly single-population GCs in our sample. As in Figure 11, median errorbars at different magnitude levels are shown in gold $(3 \sigma)$ and red $(1 \sigma)$. Unfortunately, the current $U$-band photometry of Palomar 14 is not deep enough to study MPs.

We show in Figure 11 three examples of $V, C_{U B I}$ CMDs of well-known GCs that were not previously studied by (Monelli et al. 2013). The first is NGC 5053, a very metalpoor GC that may be associated with the disrupting Sgr dwarf galaxy (although the association is debated: Tang et al. 2018). It has one of the narrowest RGBs (another such case is NGC 4590), especially if compared with similarly metal-poor GCs such as M 15, but it still covers $\simeq 0.1 \mathrm{mag}$, which is much wider than the typical errors of our photometry (Section 3, Figure 11). NGC 1261 is a metal-intermediate GC, with a bifurcated sub-giant branch (Kravtsov et al. 2010) and a diffuse stellar halo similar to that observed around NGC 1851 (Kuzma, Da Costa \& Mackey 2018). It has low field contamination because of its high Galactic latitude and therefore it shows very clean and well-defined sequences. In the $V, C_{U B I}$ plane it clearly shows a wide and possibly bimodal RGB, suggesting more a normal C-N anticorrelation rather than a complexity comparable to that of NGC 1851, but further analysis is required. NGC 7089 (M 2) is an anomalous GC like NGC 1851 but its $V, C_{U B I}$ CMD appears only slightly more complex than that of NGC 1261 and certainly not as complex as that of NGC 1851 .

One thing that we noted, that can be also seen by comparing the CMDs presented in Figures 10 and 11, is that the substructure of the RGB is definitely clearer in the $V$, $U-B$ plane, because $C_{U B I}$ is the combination of three magnitudes, with the accompanying error propagation. Moreover, the combination of the $U-B$ colour, mostly affected by $\mathrm{N}$ variations, with $B-I$, mostly affected by $\mathrm{C}+\mathrm{N}+\mathrm{O}$, helium, and iron variations, does not represent the chemistry of the populations as accurately as one would desire. Therefore, to study multiple populations with ground-based photometry it might be better to create a ground-based equivalent of the HST chromosome maps (Milone et al. 2017), i.e., a rectified colour-colour diagram of RGB stars, rather than combining the two colours in a single index.

\subsubsection{Single-population clusters}

Finding even one GC that certainly does not host MPs would be very important to define the boundaries of the MP phenomenon in the space of GC global properties like mass, age, metallicity, orbit, structural parameters, and so on. According to Carretta et al. (2010) or Carrera \& Martínez-Vázquez (2013), there seems to be a minimum cluster mass that allows for the presence of MPs, of about $10^{4} \mathrm{M}_{\odot}$. This could explain why open clusters generally do not show MPs (but see Pancino 2018) and why intermediate-age clusters in the Magellanic Clouds do (Martocchia et al. 2018). Additionally, Caloi \& D'Antona (2011) proposed a list of GCs that might contain (or be dominated by) only one population, based on their HB morphology.

However, most of the candidate single-population GCs proposed so far have been shown to actually contain MPs when studied in sufficient detail. Some recent examples are NGC 5634, which was shown to contain the Na-O anticorrelation by Carretta et al. (2017); NGC 6535, probably the lowest-mass GC to show anti-correlations (Bragaglia et al. 2017); and NGC 7099 (O'Malley \& Chaboyer 2018). NGC 6101, proposed as candidate in the past, was shown to host MPs with HST photometry (Milone et al. 2017). Very recently, another single-population candidate, IC 4499, was found to host MPs employing new, high-quality photometry and spectroscopy data (Dalessandro et al. 2018).

The GCs that still stand as single-population (or mostly single-population) candidates are the ones for which the data are presently unsatisfactory. For example, Palomar 1 had only 4 stars spectroscopically analyzed (Sakari et al. 2011); NGC 5694 only 6 stars (Mucciarelli et al. 2013); Terzan 7 only 5 stars (Sbordone et al. 2005); Terzan 814 stars with only one having high $\mathrm{Na}$ and low O (Carretta et al. 2014); Palomar 12 was studied with high-resolution (4 stars, Cohen 2004) and low-resolution spectroscopy (23 stars with 
low S/N ratio, Pancino et al. 2010), showing only hints of a bimodal C-N anti-correlation; and Ruprecht 106 had only 9 stars analyzed spectroscopically (Villanova et al. 2013) and shows evidence of a relatively small, but non-negligible, widening of the RGB according to Dotter et al. (2018).

Among the GCs in our sample, a few are still suspected of being single-population or mostly single-population GCs: NGC 5694, Terzan 8, and Pal 14 (Figure 12). Unfortunately, the current $U$-band photometry of Palomar 14 is not deep enough to draw any firm conclusion, and we will try to gather more $U$-band data in the future. While no clear multimodality can be discerned for Terzan 8 and NGC 5694 without a deeper analysis, the RGBs of these GCs are as wide as those observed in other GCs in the sample, in particular they are as wide as that of IC 4499: the typical width ranges from 0.05 to $0.15 \mathrm{mag}$, while the typical propagated $C_{U B I}$ photometric uncertainties for red giants are of the order of 0.01-0.03 mag.

To conclude, we note that even the GCs that show the thinnest RGBs in the $V, U-B$ or $V, C_{U B I}$ diagrams (such as NGC 4590, NGC 5053, IC 4499) generally have an RGB width that is at least a few times the typical photometric errors. Therefore it is safe to say that none of the GCs studied here can be classified as a genuine single-population GC, with the possible exception of a few GCs whose photometry is difficult to interpret without further analysis because of differential reddening or high field contamination.

\section{SUMMARY AND CONCLUSIONS}

We have presented wide-field, Johnson-Cousins multiband photometry and astrometry for 48 galactic GCs, based on a collection of $93272^{11}$ public and proprietary images, calibrated on the basis of 61514 local secondary standard stars (Section 2). The full data credits and observing information are provided in Appendix A. The internal and external calibration uncertainties are estimated to be of the order of a few millimagnitudes, depending on the GC and the photometric band as discussed in Section 3.1 and Appendix B. Our error estimates are confirmed by extensive literature comparisons and the residual $\mathrm{ZP}$ variations in the outer parts of the photometry are of order 0.04 mag at most (Section 3). For several GCs in the sample, these are the first publicly available photometric catalogues in the literature, at least in some of the photometric bands, typically $U$ and $R$. In particular, among those in our sample, NGC 6760 is the GC that was least studied in the past.

We explore the photometry to illustrate its scientific potential (Section 5) and find that:

- the multiband photometry and the quality indicators provided allow for a tailored selection of stars depending on the science goals of different scientific studies; the majority of contaminating field stars can be removed not only with radial selections from the GC centres, but also using colourcolour plots; the catalogues provide quality indicators that

11 They are 84106 images in UBVRI and 9166 in other bands, that were used only to improve on star's positions and deblending. allow for further cleaning of the sample from stars with significant PSF-fitting residuals or with a high chance of being variables;

- the number density and surface brightness profiles that can be obtained from our catalogues cover a large range of distances from the GC centers and provide information as detailed as the one found in the available literature studies; they will be computed for all the GCs in our database, i.e., eventually $\simeq 80 \%$ of the Harris (2010) catalogue;

- a study of the morphology of NGC 5904 showed that we can derive radial ellipticity profiles as accurate as those available in the literature, but more detailed, thanks to the wide field and depth of our photometry; we observe also the variation of the position angle of the elliptical contours with more detail (i.e., more radial bins) than previous studies;

- given the diversity of the collected photometric data in terms of photometric depth, area coverage, measurement quality, and filter bandpasses (especially $U$ ), we strongly recommend going through the image credits and sources in Appendix A to identify the exact characteristics of the data, and to allow for millimagnitude-scale residual uncertainties in our calibration of the data to the Landolt system when only a few instrumental setups are represented;

- we show that none of the GCs in our sample can be safely assumed to be free from MPs, except for a few GCs for which no clear conclusion can be made because they have significant differential reddening, field contamination, or insufficient $U$-band photometric depth; in particular, two GCs that were considered good single-population or mostly single-population candidates, Terzan 8 and NGC 5694, indeed have RGB widths that are significantly wider than expected from photometric errors alone.

Our photometry can bridge the gap between highspatial-resolution studies carried out with the HST and large photometric surveys like Gaia, SDSS, or LSST, for which no PSF photometry was performed to study the crowded GC stellar fields. Combining HST, large surveys, and our photometry with astrometry from Gaia and spectroscopy from large surveys will allow GC research to enter the Gaia era with a complete set of measurements to tackle the numerous open problems and stimulate further theoretical advancements.

\section{ACKNOWLEDGMENTS}

We warmly thank the following colleagues for interesting discussions and suggestions: P. Bianchini, R. Contreras, E. Dalessandro, V. Hénault-Brunet, C. Lardo, C. E. MartínezVázquez, S. Marinoni, P. Marrese, L. Monaco, R. M. Murillo, A. Sollima, A. L. Varri, and M. Zoccali. AZ acknowledges support through a ESA Research Fellowship. We thank the International Space Science Institute (ISSI, Bern, $\mathrm{CH}$ ) for welcoming the activities of the Team 407 "Globular Clusters in the Gaia Era". The full image sources and credits can be found in Appendix A. This research has made ample use of the Simbad astronomical database (Wenger et al. 2000) and the Vizier catalogue access tool (Ochsenbein, Bauer \& Marcout 2000), both operated at the CDS in Strasbourg, and of Topcat (Taylor 2005). Figures in this paper were produced either using the $\mathrm{R}$ programming language (R Core Team 2017; Dowle \& Srinivasan 2017) 
and Rstudio (https://www.rstudio.com/), or with Python (https://www.python.org/) and specifically with the packages numpy, scipy, and matplotlib. This work presents results from the European Space Agency (ESA) space mission Gaia. Gaia data are being processed by the Gaia Data Processing and Analysis Consortium (DPAC). Funding for the DPAC is provided by national institutions, in particular the institutions participating in the Gaia Multi-Lateral Agreement (MLA). This research has made use of the GaiaPortal catalogues access tool, ASI - Space Science Data Center, Rome, Italy (http://gaiaportal.ssdc.asi.it).

\section{REFERENCES}

Abolfathi B. et al., 2018, ApJS, 235, 42

Alvarado F., Wenderoth E., Alcaino G., Liller W., 1995, AJ, 109, 1169

Alves D. R., Bond H. E., Onken C., 2001, AJ, 121, 318

An D. et al., 2008, ApJS, 179, 326

Andersen M. I., Freyhammer L., Storm J., 1995, in European Southern Observatory Conference and Workshop Proceedings, Vol. 53, Calibrating and Understanding HST and ESO Instruments, Benvenuti P., ed., p. 87

Anderson J. et al., 2008, AJ, 135, 2055

Barmby P., McLaughlin D. E., Harris W. E., Harris G. L. H., Forbes D. A., 2007, AJ, 133, 2764

Bastian N., Lamers H. J. G. L. M., de Mink S. E., Longmore S. N., Goodwin S. P., Gieles M., 2013, MNRAS, 436, 2398

Bastian N., Lardo C., 2018, ARA\&A, 56, 83

Bellazzini M., 2007, A\&A, 473, 171

Bellazzini M., Ferraro F. R., Ibata R., 2002, AJ, 124, 915

Bellazzini M., Fusi Pecci F., Montegriffo P., Messineo M., Monaco L., Rood R. T., 2002, AJ, 123, 2541

Bellazzini M., Pancino E., Ferraro F. R., 2005, A\&A, 435, 871

Bellini A., Bianchini P., Varri A. L., Anderson J., Piotto G., van der Marel R. P., Vesperini E., Watkins L. L., 2017, ApJ, 844, 167

Bellini A. et al., 2009, A\&A, 493, 959

Bergbusch P. A., 1996, AJ, 112, 1061

Bergbusch P. A., Stetson P. B., 2009, AJ, 138, 1455

Bianchini P., van der Marel R. P., del Pino A., Watkins L. L., Bellini A., Fardal M. A., Libralato M., Sills A., 2018, ArXiv e-prints

Boberg O. M., Vesperini E., Friel E. D., Tiongco M. A., Varri A. L., 2017, ApJ, 841, 114

Bolte M., 1992, ApJS, 82, 145

Braga V. F. et al., 2018, AJ, 155, 137

Bragaglia A., Carretta E., D'Orazi V., Sollima A., Donati P., Gratton R. G., Lucatello S., 2017, A\&A, 607, A44

Brogaard K. et al., 2012, A\&A, 543, A106

Buonanno R., Corsi C. E., Ferraro I., Fusi Pecci F., 1987, A\&AS, 67,327

Caloi V., D'Antona F., 2011, MNRAS, 417, 228

Carrera R., Martínez-Vázquez C. E., 2013, A\&A, 560, A5

Carretta E., Bragaglia A., Gratton R., Lucatello S., 2009a, A\&A, 505,139

Carretta E., Bragaglia A., Gratton R. G., D’Orazi V., Lucatello S., Sollima A., 2014, A\&A, 561, A87

Carretta E. et al., 2009b, A\&A, 505, 117

Carretta E., Bragaglia A., Gratton R. G., Recio-Blanco A., Lucatello S., D'Orazi V., Cassisi S., 2010, A\&A, 516, A55

Carretta E., Bragaglia A., Lucatello S., D'Orazi V., Gratton R. G., Donati P., Sollima A., Sneden C., 2017, A\&A, 600, A118

Chen C. W., Chen W. P., 2010, ApJ, 721, 1790

Cohen J. G., 2004, AJ, 127, 1545
Cohen R. E., Sarajedini A., Kinemuchi K., Leiton R., 2011, ApJ, 727,9

Cordero M. J., Hénault-Brunet V., Pilachowski C. A., Balbinot E., Johnson C. I., Varri A. L., 2017, MNRAS, 465, 3515

Cote P., Welch D. L., Fischer P., Gebhardt K., 1995, ApJ, 454, 788

Da Costa G. S., 2012, ApJ, 751, 6

Dalessandro E. et al., 2018, ArXiv e-prints

Danilov V. M., Seleznev A. F., 1994, Astronomical and Astrophysical Transactions, 6,85

Davoust E., Prugniel P., 1990, A\&A, 230, 67

de la Fuente Marcos R., de la Fuente Marcos C., Moni Bidin C., Ortolani S., Carraro G., 2015, A\&A, 581, A13

Decressin T., Meynet G., Charbonnel C., Prantzos N., Ekström S., 2007, A\&A, 464, 1029

Denisenkov P. A., Denisenkova S. N., 1989, Astronomicheskij Tsirkulyar, 1538, 11

D'Ercole A., Vesperini E., D'Antona F., McMillan S. L. W., Recchi S., 2008, MNRAS, 391, 825

Di Cecco A. et al., 2013, AJ, 145, 103

Dotter A., Milone A. P., Conroy C., Marino A. F., Sarajedini A., 2018, ApJ, 865, L10

Dowle M., Srinivasan A., 2017, data.table: Extension of 'data.frame'. R package version 1.10.4-3

Drukier G. A., Slavin S. D., Cohn H. N., Lugger P. M., Berrington R. C., Murphy B. W., Seitzer P. O., 1998, AJ, 115, 708

Elson R. A. W., Fall S. M., Freeman K. C., 1987, ApJ, 323, 54

Fabricius M. H. et al., 2014, ApJ, 787, L26

Ferraro F. R., Carretta E., Corsi C. E., Fusi Pecci F., Cacciari C., Buonanno R., Paltrinieri B., Hamilton D., 1997, A\&A, 320, 757

Ferraro F. R., Clementini G., Fusi Pecci F., Buonanno R., Alcaino G., 1990, A\&AS, 84, 59

Ferraro F. R., Sollima A., Pancino E., Bellazzini M., Straniero O., Origlia L., Cool A. M., 2004, ApJ, 603, L81

Feuillet D. K., Paust N. E. Q., Chaboyer B., 2014, PASP, 126, 733

Gaia Collaboration et al., 2016a, A\&A, 595, A2

Gaia Collaboration et al., 2018, ArXiv e-prints

Gaia Collaboration et al., 2016b, A\&A, 595, A1

Geffert M., Maintz G., 2000, A\&AS, 144, 227

Gerashchenko A. N., Kadla Z., 2004, Astronomical and Astrophysical Transactions, 23, 35

Geyer E. H., Hopp U., Nelles B., 1983, A\&A, 125, 359

Gratton R. G., Carretta E., Bragaglia A., 2012, A\&ARv, 20, 50

Guarnieri M. D., Bragaglia A., Fusi-Pecci F., 1993, A\&AS, 102, 397

Han C., Ryden B. S., 1994, ApJ, 433, 80

Harris W. E., 1996, AJ, 112, 1487

Harris W. E., 2010, ArXiv e-prints

Hilker M., 2006, A\&A, 448, 171

Hiltner W. A., 1962, Astronomical techniques. Chicago, University Press [1962]

Holland S., Harris W. E., 1992, AJ, 103, 131

Kaluzny J., Kubiak M., Szymanski M., Udalski A., Krzeminski W., Mateo M., Stanek K. Z., 1998, A\&AS, 128, 19

Koch A., Odenkirchen M., Grebel E. K., Caldwell J. A. R., 2004, Astronomische Nachrichten, 325, 299

Kontizas E., Kontizas M., Sedmak G., Smareglia R., 1989, AJ, 98, 590

Kraft R. P., 1994, PASP, 106, 553

Kravtsov V., Alcaíno G., Marconi G., Alvarado F., 2009, A\&A, 497,371

Kravtsov V., Alcaíno G., Marconi G., Alvarado F., 2010, A\&A, 516, A23

Kravtsov V., Alcaíno G., Marconi G., Alvarado F., 2014, ApJ, 783,56 
Kravtsov V., Ipatov A., Samus N., Smirnov O., Alcaino G., Liller W., Alvarado F., 1997, A\&AS, 125, 1

Küpper A. H. W., Kroupa P., Baumgardt H., Heggie D. C., 2010, MNRAS, 407, 2241

Kuzma P. B., Da Costa G. S., Mackey A. D., 2018, MNRAS, 473, 2881

Landolt A. U., 1973, AJ, 78, 959

Landolt A. U., 1992, AJ, 104, 340

Lanzoni B., Dalessandro E., Ferraro F. R., Miocchi P., Valenti E., Rood R. T., 2007, ApJ, 668, L139

Lanzoni B. et al., 2018, ArXiv e-prints

Lardo C., Bellazzini M., Pancino E., Carretta E., Bragaglia A., Dalessandro E., 2011, A\&A, 525, A114

Lardo C. et al., 2012, A\&A, 541, A141

Lardo C. et al., 2013, MNRAS, 433, 1941

Lee J.-W., Carney B. W., 1999, AJ, 117, 2868

Lee Y.-W., Joo J.-M., Sohn Y.-J., Rey S.-C., Lee H.-C., Walker A. R., 1999, Nature, 402, 55

Lewis M. S., Liu W. M., Paust N. E. Q., Chaboyer B., 2006, AJ, 131, 2538

Libralato M., Bellini A., Bedin L. R., Piotto G., Platais I., KisslerPatig M., Milone A. P., 2014, A\&A, 563, A80

Lynga G., 1996, A\&AS, 115, 297

Manfroid J., Selman F., Jones H., 2001, The Messenger, 104, 16

Marconi G., Andreuzzi G., Pulone L., Cassisi S., Testa V., Buonanno R., 2001, A\&A, 380, 478

Marino A. F. et al., 2012a, ApJ, 746, 14

Marino A. F., Milone A. P., Piotto G., Villanova S., Bedin L. R., Bellini A., Renzini A., 2009, A\&A, 505, 1099

Marino A. F. et al., 2012b, A\&A, 541, A15

Marino A. F., Villanova S., Piotto G., Milone A. P., Momany Y., Bedin L. R., Medling A. M., 2008, A\&A, 490, 625

Martocchia S. et al., 2018, MNRAS, 473, 2688

McLaughlin D. E., Anderson J., Meylan G., Gebhardt K., Pryor C., Minniti D., Phinney S., 2006, ApJS, 166, 249

Melbourne J., Sarajedini A., Layden A., Martins D. H., 2000, AJ, 120,3127

Mészáros S. et al., 2015, AJ, 149, 153

Milone A. P., Marino A. F., Mastrobuono-Battisti A., Lagioia E. P., 2018, MNRAS, 479, 5005

Milone A. P. et al., 2013, ApJ, 767, 120

Milone A. P., Piotto G., Bedin L. R., Cassisi S., Anderson J., Marino A. F., Pietrinferni A., Aparicio A., 2012, A\&A, 537, A77

Milone A. P. et al., 2017, MNRAS, 464, 3636

Miocchi P. et al., 2013, ApJ, 774, 151

Mochejska B. J., Kaluzny J., Thompson I., Pych W., 2002, AJ, 124,1486

Momany Y., Cassisi S., Piotto G., Bedin L. R., Ortolani S., Castelli F., Recio-Blanco A., 2003, A\&A, 407, 303

Monaco L., Pancino E., Ferraro F. R., Bellazzini M., 2004, MNRAS, 349, 1278

Monelli M. et al., 2013, MNRAS, 431, 2126

Mucciarelli A., Bellazzini M., Catelan M., Dalessandro E., Amigo P., Correnti M., Cortés C., D’Orazi V., 2013, MNRAS, 435, 3667

Nardiello D., Milone A. P., Piotto G., Marino A. F., Bellini A., Cassisi S., 2015, A\&A, 573, A70

Noyola E., Gebhardt K., 2006, AJ, 132, 447

Noyola E., Gebhardt K., Bergmann M., 2008, ApJ, 676, 1008

Ochsenbein F., Bauer P., Marcout J., 2000, A\&AS, 143, 23

Odewahn S. C., Bryja C., Humphreys R. M., 1992, PASP, 104, 553

O'Malley E. M., Chaboyer B., 2018, ApJ, 856, 130

Ortolani S., Gratton R., 1990, A\&AS, 82, 71

Pancino E., 2018, ArXiv e-prints

Pancino E., Ferraro F. R., Bellazzini M., Piotto G., Zoccali M., 2000, ApJ, 534, L83
Pancino E., Rejkuba M., Zoccali M., Carrera R., 2010, A\&A, 524, A44

Pancino E. et al., 2017, A\&A, 601, A112

Pancino E., Seleznev A., Ferraro F. R., Bellazzini M., Piotto G., 2003, MNRAS, 345, 683

Pickles A., Depagne É., 2010, PASP, 122, 1437

Piotto G. et al., 2002, A\&A, 391, 945

Piotto G. et al., 2015, AJ, 149, 91

Pollard D. L., Sandquist E. L., Hargis J. R., Bolte M., 2005, ApJ, 628,729

R Core Team, 2017, R: A Language and Environment for Statistical Computing. R Foundation for Statistical Computing, Vienna, Austria

Rees, Jr. R. F., 1993, AJ, 106, 1524

Renzini A. et al., 2015, MNRAS, 454, 4197

Rey S.-C., Lee Y.-W., Ree C. H., Joo J.-M., Sohn Y.-J., Walker A. R., 2004, AJ, 127, 958

Rey S.-C., Yoon S.-J., Lee Y.-W., Chaboyer B., Sarajedini A., 2001, AJ, 122, 3219

Rosenberg A., Aparicio A., Saviane I., Piotto G., 2000a, A\&AS, 145,451

Rosenberg A., Piotto G., Saviane I., Aparicio A., 2000b, A\&AS, 144,5

Saha A., Dolphin A. E., Thim F., Whitmore B., 2005, PASP, 117, 37

Sakari C. M., Venn K. A., Irwin M., Aoki W., Arimoto N., Dotter A., 2011, ApJ, 740, 106

Samus N., Ipatov A., Smirnov O., Kravtsov V., Alcaino G., Liller W., Alvarado F., 1995, A\&AS, 112, 439

Sarajedini A. et al., 2007, AJ, 133, 1658

Sarajedini A., Milone A. A. E., 1995, AJ, 109, 269

Sariya D. P., Yadav R. K. S., 2015, A\&A, 584, A59

Sariya D. P., Yadav R. K. S., Bellini A., 2012, A\&A, 543, A87

Sbordone L., Bonifacio P., Marconi G., Buonanno R., Zaggia S., 2005, A\&A, 437, 905

Sbordone L., Salaris M., Weiss A., Cassisi S., 2011, A\&A, 534, A9

Smecker-Hane T. A., Stetson P. B., Hesser J. E., Lehnert M. D., 1994, AJ, 108, 507

Sollima A., Ferraro F. R., Pancino E., Bellazzini M., 2005, MNRAS, 357, 265

Stetson P. B., 1979, AJ, 84, 1056

Stetson P. B., 1987, PASP, 99, 191

Stetson P. B., 1989, in V Escola Avançada de Astrofisica, Barbuy B., Magalháes A. M., Janot Pacheco E., Viegas S. M., eds., Universidade de São Paulo

Stetson P. B., 1992, in Astronomical Society of the Pacific Conference Series, Vol. 25, Astronomical Data Analysis Software and Systems I, Worrall D. M., Biemesderfer C., Barnes J., eds., p. 297

Stetson P. B., 1994, PASP, 106, 250

Stetson P. B., 2000, PASP, 112, 925

Stetson P. B., 2005, PASP, 117, 563

Stetson P. B., 2009, in IAU Symposium, Vol. 258, The Ages of Stars, Mamajek E. E., Soderblom D. R., Wyse R. F. G., eds., pp. $197-208$

Stetson P. B., Bruntt H., Grundahl F., 2003, PASP, 115, 413

Stetson P. B., Catelan M., Smith H. A., 2005, PASP, 117, 1325

Stetson P. B., Fiorentino G., Bono G., Bernard E. J., Monelli M., Iannicola G., Gallart C., Ferraro I., 2014, PASP, 126, 616

Stetson P. B., Harris W. E., 1988, AJ, 96, 909

Stetson P. B., Hesser J. E., Smecker-Hane T. A., 1998, PASP, 110,533

Tang B. et al., 2018, ApJ, 855, 38

Taylor M. B., 2005, in Astronomical Society of the Pacific Conference Series, Vol. 347, Astronomical Data Analysis Software and Systems XIV, Shopbell P., Britton M., Ebert R., eds., p. 29 
Thomson G. S. et al., 2012, MNRAS, 423, 2901

Tody D., 1986, in Proc. SPIE, Vol. 627, Instrumentation in astronomy VI, Crawford D. L., ed., p. 733

Tody D., 1993, in Astronomical Society of the Pacific Conference Series, Vol. 52, Astronomical Data Analysis Software and Systems II, Hanisch R. J., Brissenden R. J. V., Barnes J., eds., p. 173

Trager S. C., King I. R., Djorgovski S., 1995, AJ, 109, 218

van den Bergh S., 2008, AJ, 135, 1731

van Leeuwen F., Le Poole R. S., Reijns R. A., Freeman K. C., de Zeeuw P. T., 2000, A\&A, 360, 472

Viaux N., Catelan M., Stetson P. B., Raffelt G. G., Redondo J., Valcarce A. A. R., Weiss A., 2013, A\&A, 558, A12

Villanova S., Geisler D., Carraro G., Moni Bidin C., Muñoz C., 2013, ApJ, 778, 186

Walker A. R., 1994, AJ, 108, 555

Walker A. R., 1998, AJ, 116, 220

Wang J. J., Chen L., Wu Z. Y., Gupta A. C., Geffert M., 2000, A\&AS, 142, 373

Wang S., Ma J., 2013, AJ, 146, 20

Watkins L. L., van der Marel R. P., Bellini A., Anderson J., 2015, ApJ, 803, 29

Welch D. L., Stetson P. B., 1993, AJ, 105, 1813

Wenger M. et al., 2000, A\&AS, 143, 9

White R. E., 1970, AJ, 75, 167

White R. E., Shawl S. J., 1987, ApJ, 317, 246

Zaritsky D., Harris J., Thompson I. B., Grebel E. K., Massey P., 2002, AJ, 123, 855

\section{APPENDIX A: DATA LOGS AND CREDITS}

As mentioned, the catalogues presented are based on 93272 individual CCD images (84 106 in filters approximating Landolt's, and another 9166 in other filters) collected over the course of more than 35 years of observation at various telescopes (Table 1).

The details of the data sources and credits are summarized in Tables A1 and A2. The former contains a full list of all the 390 observing runs used for the present study, including the program IDs and, whenever known, the name of the observer. The latter lists, for each GC, the runs from which the data are taken and the number of images used in each of the five filters. The full tables can be found in the electronic version of the journal and at CDS, here we report the list of columns and a brief description of their content.

\section{APPENDIX B: PHOTOMETRIC CALIBRATION}

Our ultimate goal is to provide final calibrated magnitudes on a photometric system as similar to that of Landolt (1992) as possible.

\section{B1 Color and extinction corrections}

It is a widely known fact that different combinations of glass filter and photosensitive detector define bandpasses that differ from camera to camera. Landolt has published throughput curves for his filters and quantum efficiency curves for his photomultipliers, but such information is not generally available for the many different equipment setups represented in our database. Other effects, such as the instantaneous state of the reflective and transmissive elements in the light
Table A1. List of all the 384 observing runs included in the present study, with some ancillary information, including the program ID or the observer, when known. The table can be found in its entirety in the online version of the journal, and at CDS.

\begin{tabular}{ll}
\hline \hline Column & Description \\
\hline Run & Unique run label (e.g., emmi8) \\
Date & Observing date(s) covered (yyyy mmm dd-dd) \\
Site & Observing site (as in Table 1) \\
Telescope & Telescope (as in Table 1) \\
Instrument & Instrument (as in Table 1) \\
$n_{U}$ & Number of images in $U$ \\
$n_{B}$ & Number of images in $B$ \\
$n_{V}$ & Number of images in $V$ \\
$n_{R}$ & Number of images in $R$ \\
$n_{I}$ & Number of images in $I$ \\
$n_{\text {other }}$ & Number of images in other filters \\
Multiplex & For mosaic cameras: \# of CCDs in mosaic \\
Program & Program ID (e.g., 083.D-0544(A), when known) \\
Observer & Observer(s) name (when known) \\
Source & Provider's name (when known) \\
\hline
\end{tabular}

Table A2. For each of the 48 GCs listed in Table 2, we report here the data sources used (from Table A1) and the number of images analyzed in each of the five UBVRI passbands, where "other" refers to non-standard passbands or other observations acquired in the same nights. The table can be found in its entirety in the online version of the journal, and at CDS.

\begin{tabular}{ll}
\hline \hline Column & Description \\
\hline Cluster & GC name as in Table 2 \\
Run & Unique run label as in Table A1 \\
Date & Observing date(s) covered (yyyy mmm dd-dd) \\
$n_{U}$ & Number of images in $U$ \\
$n_{B}$ & Number of images in $B$ \\
$n_{V}$ & Number of images in $V$ \\
$n_{R}$ & Number of images in $R$ \\
$n_{I}$ & Number of images in $I$ \\
$n_{\text {other }}$ & Number of images in other filters \\
Multiplex & For mosaic cameras: \# of CCDs \\
\hline
\end{tabular}

path of the telescope and instrument, and the instantaneous transparency of the terrestrial atmosphere, can also be important and are not known to us in advance of the data analysis.

Following standard practice that dates back at least to Johnson's and Hardie's definitive publications (pages 157 and 178 in Hiltner 1962, respectively) we compute a numerical mapping of the instrumental photometric system of each night's observing to the fundamental photometric system based upon the known broad-band colors of the predefined photometric standards. The approach exploits the fact that the family of stellar spectral energy distributions for normal stars is close to a one-parameter family driven primarily by effective temperature and reddening, and only slightly modulated by other effects such as surface gravity, chemical abundance, and rotation. A typical example of one of our transformation equations is:

$$
v=V+z_{V}+\alpha_{V} \cdot(B-V)+\beta_{V} \cdot(B-V)^{2}+k_{V} \cdot X
$$


where $v$ is the instrumental magnitude obtained during the course of our measurements, which scales as $-2.5 \log [$ (counts)/(integration time)]; $V$ is the standard visual magnitude as published by Landolt (1992); $z_{V}$ is a photometric ZP which accounts for the overall transmission of the telescope and instrument optics, filter throughput, and detector quantum efficiency; $B-V$ is the star's measured color, again as published by Landolt (1992); $X$ is the airmass at which the observation is obtained, such that the airmass is defined to be unity when observing at the zenith and goes very nearly as $\sec z$, where $z$ is the angular zenith distance $^{12}$. In this equation, the quantities $V, B-V$, and $X$ are presumed to be known quantities with uncertainties negligible compared to the uncertainty of the observed quantity $v$, while $\alpha_{V}, \beta_{V}$, and $k_{V}$ are treated as quantities that are, initially, completely unknown and must be derived empirically from our own observations of $v$ for many diverse stars by the method of (robust) least squares. Very similar equations are also employed for the $U, B, R$, and $I$ photometric bandpasses, with some detailed modifications to be discussed below.

In this calibration, we take cognizance of the fact that the $v$ bandpass defined by the total throughput of our combination of atmosphere, telescope, and filter multiplied by detector sensitivity, as a function of wavelength $(\lambda)$ is not identical in detail to Landolt's $V$ bandpass. We ask the question: when Landolt observes a star to have a particular $V$ and $B-V$, what $v$ magnitude do we observe? The calibration equation illustrated above represents a second-order Taylorexpansion answer to that question, where $B-V$ takes on the rôle of $\mathrm{d} \log ($ flux $) / \mathrm{d} \lambda$ and $\alpha$ and $\beta$ are an empirical secondorder description of the $\Delta \lambda$ and $\Delta \lambda^{2}$ differences between Landolt's $V$ photometric bandpass and our $v$ bandpass.

In the case of the $B$ filter, we add a term in the calibration equal to $-0.016(B-V) X$, to correct in gross fashion for the fact that hot blue stars preferentially produce photons that pass through the shorter-wavelength flank of the $B$ filter, while more of the light from red stars passes through the longer-wavelength side of the filter. The difference in the effective wavelength of the $B$ filter for stars of extreme color is sufficient that the hotter stars experience perceptibly higher extinction in the terrestrial atmosphere than cooler stars. The value -0.016 is obtained by a numerical analysis of observed stellar spectral-energy distributions multiplied by Landolt's $B$ bandpass and a model terrestrial atmosphere for an observatory roughly $2000 \mathrm{~m}$ above sea level. The effect is too subtle to be measured with any precision in our actual photometric data.

The color terms in $B-V$ are replaced by corresponding terms in $V-R$ in the equation for the $R$ band, and by terms in $V-I$ for the $I$ equation. This ensures that the gross slope of the spectral energy distribution is estimated at wavelengths close to the bandpass being considered.

The $U$ bandpass represents a particular problem. Landolt's $U$ bandpass is defined on the short-wavelength side by the throughput of the terrestrial atmosphere more than by either filter throughput or detector sensitivity. Furthermore, the $U$ filter as provided by most observatories is by

12 In fact, a more accurate formula for $X$ that takes into account the curvature of the atmosphere is employed, but the distinction is not important for this discussion. far the most poorly standardized. Finally, the $U$ bandpass contains the Balmer convergence and jump, as well as significant metal-line blanketing and molecular bands, including most especially the $388 \mathrm{~nm}$ band of CN. Thus, unlike the case with the other filters, the $U$ magnitude is not a monotonic function of temperature for fixed apparent $V$ or other reference magnitude. Our default calibration equation for our instrumental $u$ observations is

$$
u=U+z_{U}+\alpha_{U} \cdot(U-B)+\beta \cdot(B-V)+\gamma_{U} \cdot(B-V)^{2}+k_{U} \cdot X .
$$

To the extent that the classical $(U-B, B-V)$ color-color diagram can be approximated by three line segments- $U-B$ increases with $B-V, U-B$ decreases as $B-V$ increases, $U-B$ increases with $B-V$-with smooth transitions between, this formulation allows the first-order Taylor expansion $u-U \sim$ $\gamma(U-B)$ to have a different slope in each of the three regimes of $B-V$. In our experience, the data are almost never good enough to determine reliable coefficients for still higher-order terms. In particular, our limited attempts to estimate a color-extinction term for $U$, similar to that employed for $B$, have not yet led to perceptible improvements in the photometry. The likelihood is that this relatively subtle effect is being swamped by grosser systematics due to the greater complexity of stellar spectral energy distributions at these wavelengths and the diversity of the available $U$-band filters.

\section{B2 Spatial corrections}

Wide-field cameras and other instruments with focal reducers often suffer from vignetting and distortion effects (Andersen, Freyhammer \& Storm 1995) that introduce variations in the measured stars' magnitudes as a function of position on the focal plane, beyond what can be corrected by the flat-field corrections. Scattered light that enters the flatfield exposures but is not present during night-time science exposures can also introduce a spurious positional gradient in the flux scale of flat-fielded science images. To the extent practical, vignetted regions were included in the data masks derived for each chip for each observing run, and so should have been largely omitted during our photometric reductions. To deal with residual photometric variations that result from marginal vignetting and imperfect flat-field exposures, we routinely add terms $+\delta x+\epsilon y$ to the calibration equations presented in the previous section, where $x$ and $y$ are the stars' coordinates in the natural pixel grid of the CCD. For mosaic cameras these coefficients are determined completely independently for the individual chips. As a result, calibration effects that are primarily radial with respect to the center of the array can be adequately approximated by effects that are planar over the individual detectors. These coefficients are also left to vary freely from night to night, as are the ZPs, under the assumption that the different flat fields obtained for different nights might not experience the same contamination.

For very small detectors, a few arcminutes on a side and perhaps not containing a large number of standard stars, these spatial terms are often omitted and any uncorrected ZP gradient contributes to the observed scatter in the photometric residuals. Conversely, for large detectors where the photometric scatter is large, we inspect residual plots and, where indicated, add calibration terms in $x^{2}, x y$, and $y^{2}$. In 
the case of the ESO WFI, Manfroid, Selman \& Jones (2001) and Koch et al. (2004) report 0.1-0.2 mag maximum variations that can be repaired with a mosaic-wide quadratic form. In fact, for the WFI images employed here, quadratic terms never led to a significant reduction in our fitting residuals, presumably because our individual linear corrections for the different chips were adequate. However, there were a few cases where quadratic positional corrections improved the residuals for large, on-axis CCDs.

\section{B3 Night-to-night differences}

Obviously, independent values of the various $\alpha$ 's, $\beta$ 's, and $\gamma$ 's, are obtained for each observing run; the $k$ 's, $\delta$ 's and $\epsilon$ 's are almost always determined independently for each individual photometric night. However, normally we will require the different CCDs of a mosaic camera to have the same value of $k$ in a given filter on a given night under the assumption that all are looking through effectively the same atmosphere during an integration ${ }^{13}$. Usually we will enforce the same value of $\beta$, and often the same value of $\alpha$ on the different CCDs of a mosaic, under the assumption that different CCDs of almost identical manufacture will have similar quantum efficiency curves, such that the photometric bandpass is mostly defined by the atmosphere, telescope, camera optics, filter, and the average CCD sensitivity dependence as a function of $\lambda$; any residual differences in, say, the overall quantum efficiency and gain of each detector, and gross positional variations in the throughput of the filter are adequately absorbed into the different detectors' ZPs, $z$ - which are always left to be freely and independently determined for each CCD on each night - and $\delta$ and $\epsilon$.

Similarly, we usually require the coefficients $\alpha$ and $\beta$ to be the same for a given detector on consecutive nights of an observing run where there is no evidence that an instrument change has taken place, under the assumption that these parameters are defined by physical properties of the equipment that should not vary on timescales of days. It also goes without saying that we examine the residuals of our transformation fits and if we see evidence that our assumptions fail to a degree that is significant compared to the random uncertainties of our observed magnitudes, we leave the corresponding parameters free in the least-squares reductions.

On nights when the observed scatter in the standardstar residuals and/or bizarre extinction coefficients imply that photometric conditions were not obtained, we do not compute extinction coefficients $k$ or nightly ZPs $z$. Instead we compute an independent photometric ZP for each individual CCD image based on the photometric standards contained within that image; if an image from a non-photometric night contains no photometric standards, it is of no scientific use to us.

13 And also assuming that the atmosphere is the only source of airmass dependence in the observation: instrumental flexure, for instance, is assumed not to be a significant factor

\section{B4 Magnitude averages}

To obtain final Landolt-system magnitudes for our target stars, that is, for stars for which these magnitudes are not known a priori, we use exactly the same transformation equations, but the terms on the right sides of the equations reverse their rôles. The instrumental magnitudes ( $v$ and $b$, for example) continue to be observed quantities which alone contain uncertainty. Also as before, the airmass $X$ is presumed to be known with perfect geometric accuracy for each observation. Unlike before, however, the $z$ 's, $\alpha$ 's, $\beta$ 's, $\gamma$ 's $\delta$ 's, $\epsilon$ 's and $k$ 's are now presumed to be known quantities, with uncertainties that are negligible compared to the observational uncertainties in $v$ and $b$. The standard-system magnitudes ( $V$ and $B$ in this example) and the implied value of $B-V$ are now the unknown quantities to be determined via least squares.

All of the available values of $v$ from all of the images on all of the nights from all of the observing runs where that star has been observed in each observatory's approximation of the $V$ filter, and likewise all of the available values of $b$ are employed in a numerical solution to derive that singular value of $V$ and that singular value of $B$ that best explain all the observations in a robust least-square sense. Since $B-V$ is not known ahead of time, a typical value must be assumed initially, and the pair of transformation equations solved iteratively. As long as $|\alpha|,|\beta|,|\gamma| \ll 1$ convergence to an unambiguous solution of the system of equations is extremely rapid. Even if $|\alpha, \beta, \gamma| \lesssim 1$, convergence occurs eventually unless few observations are available and some of them are highly defective. This approach presumes, of course, that the star is not variable on time scales relevant to the available data, but the root-mean-square residuals of the solutions to these equations is always computed and is available as an index of intrinsic variability or photometric blunder. Stars with repeatability poorer than expected from the estimated uncertainties of the individual values of $v$ and $b$ are flagged and identified as either candidate variable stars or stars with seriously defective observations.

Overall, our network of calibration equations is both empirical and approximate, necessarily, given the nature of the data and the problem. For average stars observed under average conditions, the calibration approaches exactitude; for extreme stars observed under extreme conditions, it is approximate. Unmodeled complexity in the color-dependent extinction beyond the corrections described here, for instance, might mean that very red stars observed at high airmass will have a residual calibration error that is of one sign, while stars much bluer than average would have a calibration error of the opposite sign. For those same stars observed at very low airmass the signs of the residual errors will reverse, and the average results, interpolated to average airmass, will be nearly correct. The behavior of the same two stars observed during a different observing run might be similar, or opposite, or indeed the residual calibration errors might be effectively zero. In the long run, over many observing runs with many equipment setups, the net results of neglected complexity in the model transformations should decay away, in the average.

These residual calibration errors will still contribute, however, to the observation-to-observation repeatability of the magnitudes for individual stars, and will therefore be 
reflected in the standard errors of the mean magnitudes that we report with each measured magnitude. In general, therefore, we expect that stars of "typical" colors, say $0.5<B-V<1.1 \mathrm{mag}$, will have very reliably determined magnitudes. Results for stars of extreme color, $B-V \lesssim 0$ or $B-V \gtrsim 2.0$, for example, will be more uncertain and will have larger reported uncertainties. Stars near the celestial poles may have larger systematic errors than stars that pass overhead, because they can never be observed at low airmass. But all these problems should (a) be reflected in our reported standard errors of the mean magnitudes for each star, and (b) decrease as the number of independent times and nights and observing runs during which the star was observed increases.

In general, our magnitudes will be least reliable in $U$, where the total number of observations is small and the diversity of available filters is large. In particular, there is the possibility of a drift in the ZP of our $U$ magnitude scale for very blue and very red stars near the two celestial poles, where our neglect of a color-extinction correction in the $U$ transformation equation becomes a systematic, rather than a random error. The magnitudes will be most reliable in $V$, where the number of available images is the largest, and the homogeneity of the photometry is high due to the relative simplicity of stellar spectral energy distributions at these wavelengths and the general similarity of the various observatories' $V$ filters. $B$ and $I$ should be nearly as good as $V$, while $R$ suffers mainly from a paucity of available data. These statements are further quantified in Section 3.1. 